\title{
Ultrafast Time-of-Flight Method of Gasoline Contamination Detection Down to ppm Levels by Means of Terahertz Time-Domain Spectroscopy
}

\author{
Kamil Stelmaszczyk ${ }^{1, * \mathbb{D}}$, Ewelina Karpierz-Marczewska ${ }^{1} \mathbb{D}^{\mathbb{D}}$, Valeri Mikhnev ${ }^{1}$, Grzegorz Cywinski ${ }^{1} \mathbb{D}$, \\ Thomas Skotnicki ${ }^{1,2,3}$ and Wojciech Knap ${ }^{1,4}$ (D)
}

Citation: Stelmaszczyk, K.; Karpierz-Marczewska, E.; Mikhnev,

V.; Cywinski, G.; Skotnicki, T.; Knap, W. Ultrafast Time-of-Flight Method of Gasoline Contamination Detection Down to ppm Levels by Means of Terahertz Time-Domain Spectroscopy. Appl. Sci. 2022, 12, 1629. https:// doi.org/10.3390/app12031629

Academic Editor: Vyacheslav A. Trofimov

Received: 2 January 2022 Accepted: 1 February 2022 Published: 4 February 2022 Corrected: 20 May 2022

Publisher's Note: MDPI stays neutral with regard to jurisdictional claims in published maps and institutional affiliations.

Copyright: (c) 2022 by the authors. Licensee MDPI, Basel, Switzerland. This article is an open access article distributed under the terms and conditions of the Creative Commons Attribution (CC BY) license (https:/ / creativecommons.org/licenses/by/ $4.0 /)$.
1 CENTERA Laboratories, Institute of High Pressure Physics Polish Academy of Sciences, ul. Sokołowska 29/37, 01-142 Warsaw, Poland; ekarpierz@unipress.waw.pl (E.K.-M.); mvaleri@unipress.waw.pl (V.M.); gc@unipress.waw.pl (G.C.); t.skotnicki@cezamat.eu (T.S.); wojciech.knap@unipress.waw.pl (W.K.)

2 CEZAMAT Centre for Advanced Materials and Technologies, Warsaw University of Technology, ul. Poleczki 19, 02-822 Warsaw, Poland

3 Institute of Microelectronics and Optoelectronics, Faculty of Electronics and Information Technology, Warsaw University of Technology, ul. Koszykowa 75, 00-662 Warsaw, Poland

4 Laboratoire Charles Coulomb, UMR, CNRS 5221, 34095 Montpellier, France

* Correspondence: kamil.stelmaszczyk@unipress.waw.pl; Tel.: +48-22-182-12-47

Featured Application: Fast (picoseconds scale) and sensitive (ppm-level) detection of impurities in gasoline employing terahertz time-domain spectroscopy systems (THz-TDS) for petrochemical production lines or quality assessment and control facilities.

\begin{abstract}
In this article, we present the possibility of using terahertz time-domain spectroscopy to detect trace, ppm-level (\%wt.) concentrations of admixtures in 95-octane lead-free gasoline in straightforward time-of-flight (pulse-delay) measurements performed directly in the liquid. The method was tested on samples containing $75-0.0125 \%$ of isopropanol and $0.3-0.0250 \%$ of water. The detection limits for isopropanol and water content were determined to be $125 \mathrm{ppm}$ and $250 \mathrm{ppm}$, respectively, approaching the limits of much more complicated and time-consuming methods (like spectroscopy of the vapor phase). The measured pulse delays were compared with theoretical calculations using the modified Gladstone-Dale mixing rule for the solutions. The comparison demonstrated good agreement for gasoline-alcohol mixtures and large discrepancies for gasolinewater mixtures, suggesting that gasoline-water mixtures cannot be considered idealized binary mixtures. Our results clearly show that the pulse time delay measurement by THz-TDS is a fast and sensitive method of gasoline contamination detection and, as such, can be easily integrated with industrial online real-time quality control applications.
\end{abstract}

Keywords: terahertz time-domain spectroscopy; terahertz industrial and nondestructive evaluation; terahertz time-of-flight methods; ppm-level detection in liquid phase; petrochemicals; gasoline fuel additives and contamination; refractive index measurements; gasoline; water; isopropanol

\section{Introduction}

Crude oil and its refined products, generally known as petrochemicals, are among the most important sources of energy, fuel and raw materials; without crude oil and petrochemicals, modern life would not be possible. Petrochemicals consist predominantly of solid, liquid and gaseous hydrocarbons which dissolve one another. The main components of petrochemicals are alkanes, cycloalkanes, aromatic hydrocarbons and, to a small extent, alkenes. The main contaminants are water, organic compounds containing oxygen, sulfur or nitrogen, as well as some inorganic compounds. In the fuel industry, both aviation fuels 
and motor fuels are obtained by refining crude oils. Thus, even in the final fuel, there are always some traces of different contaminants, including water [1].

Water is a particularly undesirable component of fuels, especially in the aviation industry. Due to its polar structure, it is almost immiscible with the organic compounds of which fuel is composed. In fuels, water is present in three forms: dissolved water, free water (a separate liquid phase) and fuel-water emulsions [2,3].

Dissolved water is not dangerous for the proper working of the engines, and it is regarded as a minor fuel constituent [3]. However, water is miscible in fuel only within a small concentration range, and its exact saturation concentration depends on the fuel's composition. For example, in the case of hydrocarbon mixtures containing higher contents of aromatics, more water can be dissolved. This occurs because water is more soluble in benzene than in other hydrocarbons.

Above a certain concentration level and depending on external conditions (temperature, pressure), water begins to separate from the solution, forming free water [1,4]. If surfactants are present in a mixture, the water and fuel form an emulsion where droplets of water less than $100 \mu \mathrm{m}$ in diameter are constantly dispersed in the fuel phase.

Both free water and emulsions are dangerous forms of water in fuels. There are many factors that make water in fuel undesirable, of which the most important are corrosion, lubrication loss and fuel flow obstruction.

Water can corrode fuel tanks, which creates a risk of contamination with solid particulates of rust or even fuel leakage. Water is less viscous than diesel and, therefore, provides a smaller "cushion" of lubrication between the opposing surfaces of the moving parts, leading to increased abrasive wear. Therefore, the presence of water deteriorates the lubricating properties of a fuel. Water will freeze at sub-zero Celsius temperatures, and the resulting ice crystals, like other solid particles, may clog fuel filters, cause mechanical damages and/or impede fuel flow. Water in the fuel may also facilitate the growth of microorganisms that can produce sludge and acids that contaminate the fuel and give rise to corrosion of the tank and fuel systems.

The multiple problems caused by water in fuels (partially those enumerated above) mean that new, cheap and quick methods to determine its exact content are constantly being sought [2,5]. One of the most intensively explored methods is terahertz time-domain spectroscopy (THz-TDS). THz-TDS is a rapidly developing spectroscopic technique often used for determining the properties of a material by probing it with short pulses of $\mathrm{THz}$ radiation [6]. THz-TDS is particularly attractive for analytical applications in a gas phase, where frequencies of molecular twisting or stretching or intramolecular or intermolecular hydrogen bonding often correspond to the $\mathrm{THz}$ range $[7,8]$.

The possibility of detecting differences in molecular configuration and polarity of hydrocarbons and other polar species, such as water or isopropanol, is strictly related to the fact that nonpolar molecules are hardly visible in the $\mathrm{THz}$ spectral range in contrast to large-dipole-moment molecules [9-11]. Therefore, based on the pulse shape and its spectrum, the composition of a sample can be determined, and the parameters, such as the refractive index or absorption coefficient as a function of $\mathrm{THz}$ radiation frequency, can be calculated.

THz-TDS has also been used as a method of detecting water or other polar compounds in petrochemicals. The published research focuses only on crude oils and diesel fuels [12-14]. Generally, the obtained sensitivities oscillate around 1\% [11,12]; however, the authors of [13] reported on extremely low detection limits of $0.01 \% \mathrm{wt}$. for their measurements. This result is rather difficult to verify because no absorption data for dehydrated samples were given in the paper as a reference. An interesting correlation between $\mathrm{THz}$ pulse delay and the amount of water contained in crude petroleum was reported in [14], but in this case, the reported water content in samples was rather high, i.e., ranging from $1.8 \%$ to $90.6 \%$.

In this article, we study the limits of sensitivity of THz-TDS detection of contamination of gasoline. We study mixtures of gasoline with water and isopropanol and show that the 
lowest detection limits for isopropanol and water content that can be achieved by using one of the best commercial THz-TDS systems are $125 \mathrm{ppm}$ and $250 \mathrm{ppm}$, respectively. These values approach those of much more complicated and time-consuming spectroscopic methods (like spectroscopy of the evaporated gasoline gas phase). The measured pulse delays are compared with theoretical calculations using the modified Gladstone-Dale mixing rule for the solutions. The comparison demonstrates good agreement for gasolinealcohol mixtures and large discrepancies for gasoline-water mixtures, suggesting that gasoline-water mixtures cannot be considered as idealized binary mixtures.

Our work clearly shows that the pulse time delay measurement by THz-TDS is a sensitive and fast method of gasoline contamination detection and, as such, can be easily integrated with industrial real-time quality control applications.

\section{Materials and Methods}

\subsection{Samples and Samples Preparation}

Two-component mixtures consisting of gasoline and deionized and distilled water or isopropanol were prepared. The isopropanol ( $\mathrm{min} .99 .7 \%$, pure PA) was bought from the Chempur company, while deionized water was obtained directly from the laboratory supply line. The gasoline, 95-octane lead-free petrol (Orlen Eurosuper 95 lead-free), was purchased from a local petrol station. According to the specifications given by the producer, the maximum oxygenate content in 95-octane lead-free gasoline, which is comprised of isopropanol and water, is smaller than $2.7 \%(\mathrm{~m} / \mathrm{m})$ [15]. This clearly shows that there was a certain unknown amount of water and isopropanol in the samples during the measurements. The time-of-flight method is only capable of detecting an excess of water/isopropanol in relation to the base amount present in gasoline. However, we have paid special attention to keep this base amount unchanged and distributed equally among the samples with decreasing concentrations of admixtures: (i) pure gasoline was freshly bought and stored at low temperature in a special safe storage container to minimize any compositional changes and reduce the influence of external humidity on the water content in gasoline; (ii) the time needed to handle the solutions (weighing, mixing, pouring, etc.) was shortened to the minimum amount necessary and was comparable for each of the samples; (iii) the samples were freshly prepared before each experimental run under a fume hood with a constant flow of clean dry air, and they were measured within a maximum of 2 days after their preparation; (iv) each of the concentrations were obtained and measured at least twice to verify the self-consistence of the obtained results; (v) during the measurements, the cuvettes were tightly sealed with gasoline-resistive caps, so that their composition did not change at the time of measurement; (vi) all samples were stirred during the preparation for at least $30 \mathrm{~min}$ and again directly before the measurements to confirm their homogeneity based on visual observation.

During all measurements, the sample containing pure gasoline served as the reference. All solutions were prepared by diluting a solution of a higher concentration with freshly added gasoline to exclude a gross error. The stock solution was weighted on a laboratory scale and mixed with water/isopropanol in the right proportions to obtain a total mass of $20 \mathrm{~g}$. The samples with lower admixture concentrations were obtained by dilution of the higher admixture concentrations. Such a procedure is well-established and commonly applied in chemistry to dissolve solutes at the ppm level of their concentration [16-18]. We are, therefore, sure that the concentrations that were obtained corresponded to the nominal concentrations.

The isopropanol-gasoline solutions were prepared over the concentration range of isopropanol from $75 \% \mathrm{wt}$. to $0.0125 \% \mathrm{wt}$. (detailed concentration data can be found in Sections 3.3 and 3.4). Due to the much lower solubility of water in gasoline, the samples containing water were prepared in a smaller range of water concentrations from $0.3 \%$ wt. to $0.025 \%$ wt. The solubility of water in the gasoline type used for the sample preparation was tested before the measurements. We found that it was possible to dissolve about $0.31 \%$ wt. 
of water in the gasoline. The upper concentration limit of water, $0.3 \%$ wt., was decided based on this result.

During all measurements, the pure gasoline sample served as the reference. All solutions were prepared by diluting a solution of a higher concentration with freshly added gasoline. Each concentration was obtained at least twice and measured. All samples were stirred directly before measurements and confirmed to be homogeneous based on visual observation.

The admixture solutions were measured in cuvettes. Standard optical spectroscopy absorbance quartz cuvettes were sufficient for most of the measurements; however, to ensure a high enough amplitude of the signal over long optical paths, cuvettes made of polymethylpentene (TPX), which are characterized by their very low absorption of $\mathrm{THz}$ radiation, were used. The quartz cuvettes were the QS-type cuvettes produced by Hellma Optik GmbH. We used cuvettes with optical paths of $l=1.00 \mathrm{~mm}, 2.00 \mathrm{~mm}$ and $5.00 \mathrm{~mm}$. The maximum accuracy of $l$ specified by the producer was as low as $\pm 0.003 \mathrm{~mm}$. The wall thickness in all cuvettes was the same: $1.25 \mathrm{~mm}$ each. The TPX cuvette was manufactured by BATOP GmbH. It was made from TPX polymer (complex refractive index $1.46+0.85 \times 10^{-3}$ ) and provided a low-loss optical path in liquid of $l=10.00 \mathrm{~mm}$. The wall thickness of the TPX cuvette was $5.00 \mathrm{~mm}$. We prepared separate caps for the two types of cuvettes and used them during the measurements in order to prevent the liquids from evaporating.

\subsection{Experimental Setups}

\subsubsection{Terahertz Time-Domain Spectrometer}

Times of flight (TOFs) of the THz pulses transmitted through gasoline samples were measured by means of a stand-alone commercial THz-TDS spectrometer from Toptica A.G. [19]. The two different quasi-optical beam delivery arrangements, $4 f$ and $2 f$, were used with the spectrometer, as depicted in Figure 1. The differences between them are described in detail in the following Section 2.2.2.

A pair of $\mathrm{THz}$ transmitter (Tx) and receiver ( $\mathrm{Rx}$ ) photoconductive antennas operating on the principle of an Auston switch were used in the spectrometer [20,21]. They employed a state-of-the-art InAlAs/InGaAs multilayer structure technology [22,23]. The optopair was excited and subsequently probed by the Erbium fiber laser of $1.56 \mu \mathrm{m}$ wavelength. The duration and repetition rate of the laser pulses were, respectively, $80 \mathrm{fs}$ and $100 \mathrm{MHz}$. On the transmitter side, the laser delivered an average power of $20 \mathrm{~mW}$, generating $30 \mu \mathrm{W}$ average $\mathrm{THz}$ power. The duration of the individual THz pulses was approx. $2 \mathrm{ps}$.

The probe pulses of the laser were delayed with respect to the excitation pulses by means of the two-stage delay line. The first one (not shown in Figure 1), the long-travel delay line, introduced a constant time offset (up to $1 \mathrm{~ns}$ ) of the pulses to compensate for the optical path length. The second delay line (shown in Figure 1), the fast delay line, provided time stamping for the precise sampling of the waveforms. Its readout was synchronized with the signal from the Rx receiver during a continuous forward and backward scan of the delay line mirror. In this way, the dead time of the system was reduced, allowing the $\mathrm{THz}$ pulse sampling to be completed in a twice shorter time. Sampling rates of approximately $12 \mathrm{pulses} / \mathrm{s}$ by time windows of $100 \mathrm{ps}$ were achievable, allowing for less than $1.5 \mathrm{~min}$ acquisition times by applied averaging over 1,000 waveforms. Due to the high-precision position sensor of this delay line, which provided $1.3 \mathrm{fs}$ and stable timing resolution, the system was characterized by the high accuracy of the time scale, resulting in a typical dynamic range of $98 \mathrm{~dB}$ when multiple traces were averaged [24]. Such a high dynamic range combined with the operational stability of the TDS spectrometer were crucial for obtaining low, ppm-level admixture detection limits during the measurements. An example of the time profiles and power spectra of the $\mathrm{THz}$ pulses registered with the spectrometer in ambient air is shown in Figure 2. 


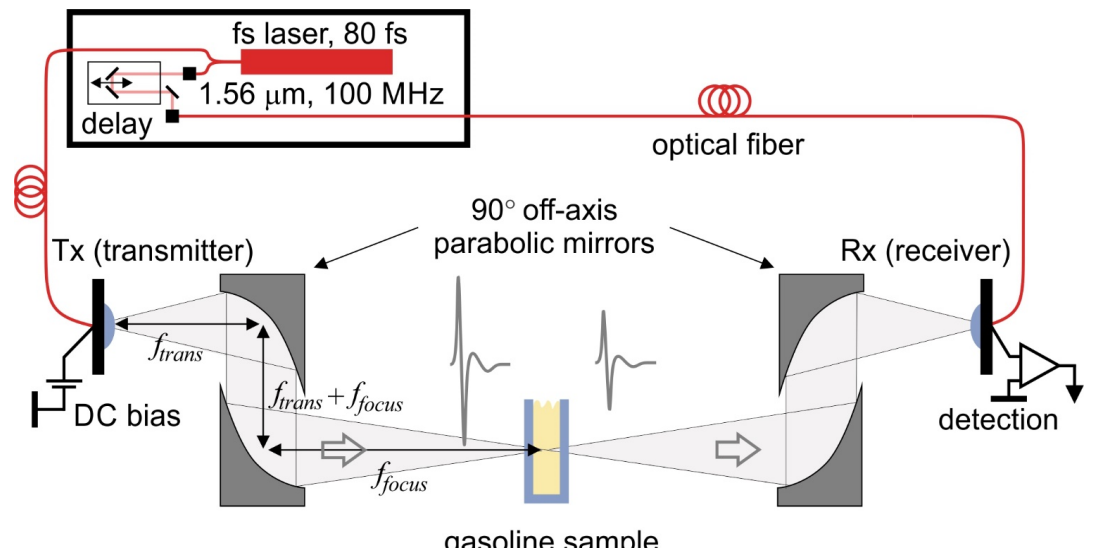

(a)

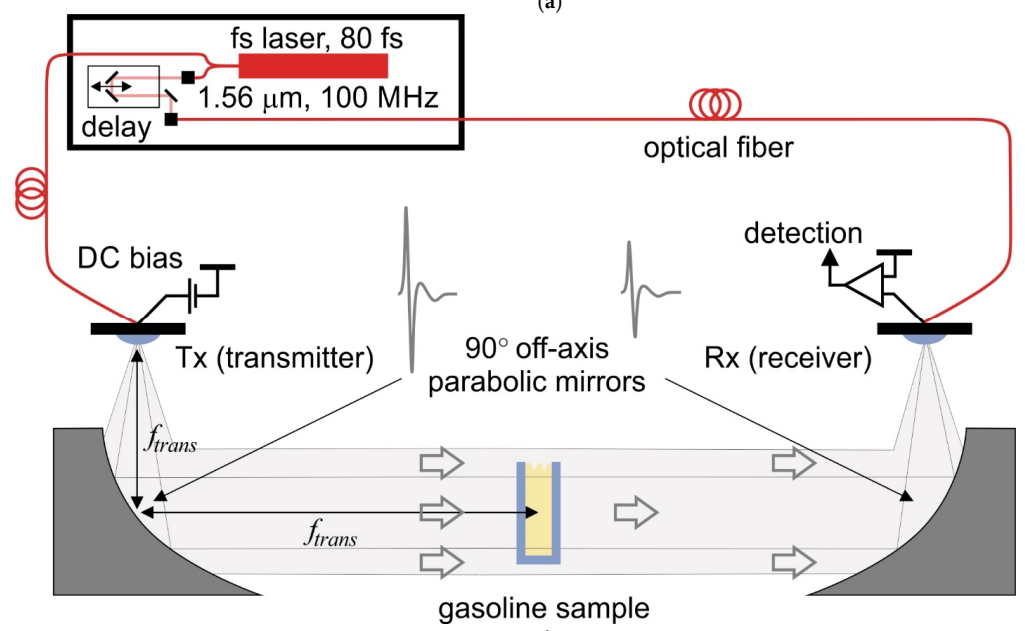

(b)

Figure 1. Schematic representation of the measurement setups: (a) $4 f$ setup consisting of four $90^{\circ}$ offaxis parabolic mirrors, which were used to measure $\mathrm{THz}$ pulse TOFs through the gasoline admixtures samples in quartz cuvettes of 1.00, 2.00, and $5.00 \mathrm{~mm}$ lengths in a focused beam configuration without a time-reference pulse; (b) $2 f$ setup consisting of two $90^{\circ}$ off-axis parabolic mirrors used for the measurements in a $10.00 \mathrm{~mm}$-long TPX cuvette with a time-reference pulse. Note that the diameter of the $\mathrm{THz}$ beam is somewhat larger than the size of the cuvette and passes freely through the inert gas, serving as a reference for the measured TOF. The indicated focal lengths are not to scale.

\subsubsection{Quasi-Optical THz Beam Delivery Setups}

As depicted in Figure 1, the two types of quasi-optical beam delivery setups were used to bring $\mathrm{THz}$ radiation to the gasoline samples and, next, onto the detector. In the $4 f$ setup (Figure 1a), the measuring cuvette was positioned in the Fourier plane of the optical system at a midway distance between the transmitter and receiver. The relationship between the Tx transmitter-emitted beam waist, $\omega_{\text {trans }}$, which is next collimated and focused down to the focused beam waist size, $\omega_{\text {focus }}$, can be deduced from the Gaussian beam theory, which is explained in detail in Appendix A.

In short, away from its source, the $\mathrm{THz}$ beam appears to diverge from the central point of the Tx transmitter, with the half-divergence angle, $\theta_{\text {trans }}$, being proportional to the wavelength of the radiation, $\lambda$, and inversely proportional to $\omega_{\text {trans }}$. For the Tx transmitter, $\omega_{\text {trans }}$ can be approximated by the radius of the photoconductive antenna lens, which defines the final output aperture of the emitted $\mathrm{THz}$ pulse. A simple proportionality relation links $\omega_{\text {trans }}$ and $\omega_{\text {focus }}$ with the focal lengths of the collimating and focusing mirrors in the $4 f$-type beam delivery setup. The formal derivation of this relation, which is expressed by Equation (A8), is given in Appendix A. 


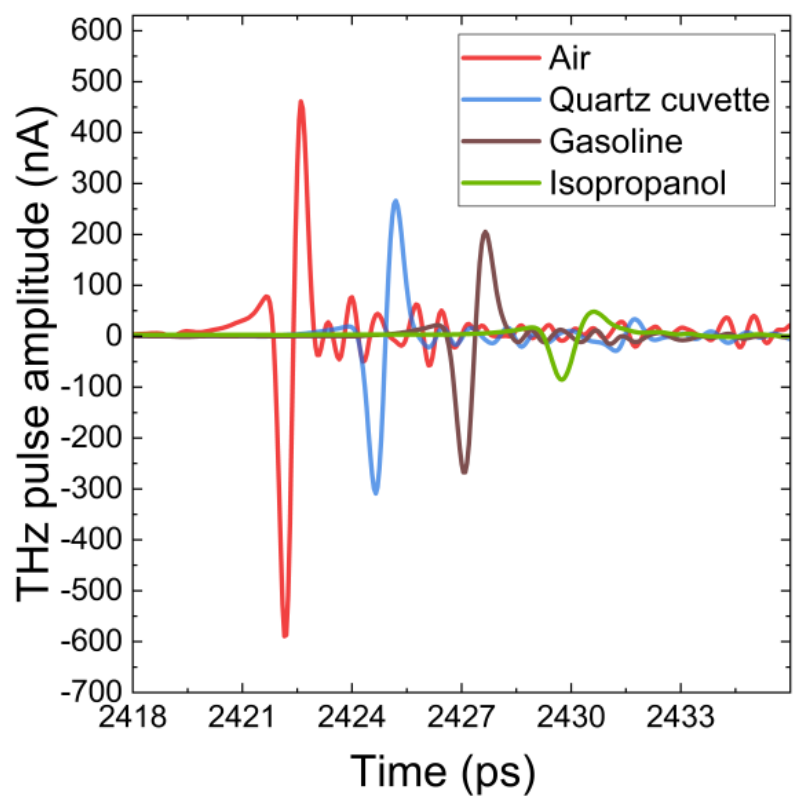

(a)

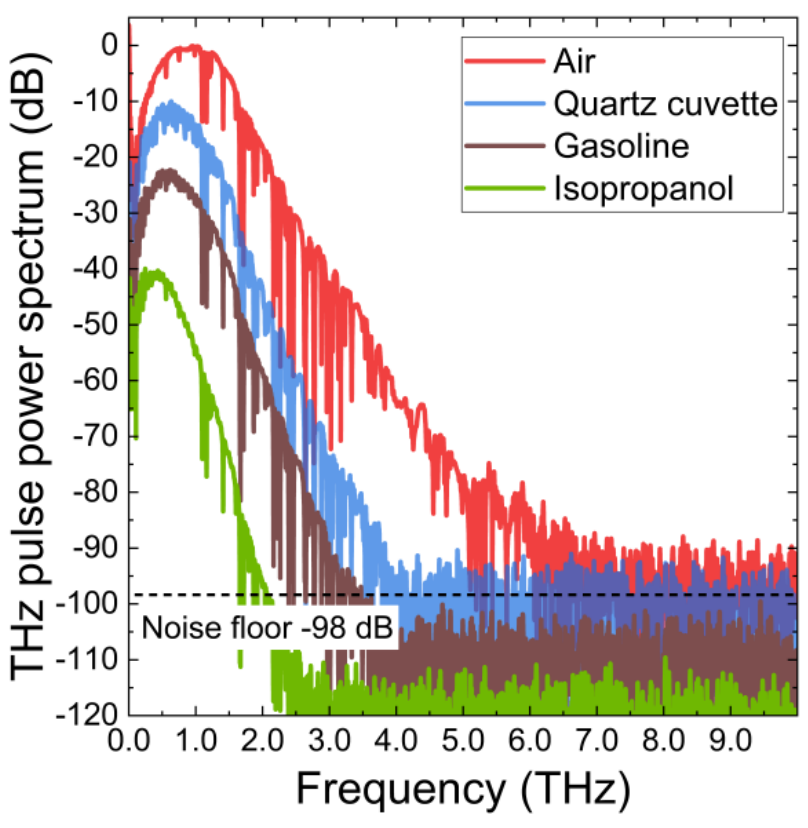

(b)

Figure 2. Performance of the THz-TDS system during measurements. Pulses were transmitted through ambient air without any sample. A $1.00 \mathrm{~mm}$-long empty quartz cuvette was used, as well as the same cuvette filled with pure gasoline and isopropanol. (a) Time-profiles of the pulses with an artificially added horizontal offset of approx. 2.5 ps. (b) Power spectra of the pulses; a vertical offset $\sim 10 \mathrm{~dB}$ was added to visualize spectral features without overlapping. The noise floor level is representative for all spectra; however, it corresponds to the "true" noise line of the spectrum registered in ambient air. The reduction of the dynamic range and bandwidth is caused by the absorptive properties of substances.

Actually, $\omega_{\text {focus }}$ is independent of the wavelength or frequency of the radiation. This is a favorable situation from the point of view of TDS measurements, assuring comparable sizes of the irradiation spots focused on the sample. A tradeoff, in this case, is the frequency-dependent Rayleigh length, $Z_{R}$, which defines the distance along which the beam propagating through the cuvette can be considered parallel to the optical axis.

When $Z_{R}$ is substantially shorter than the cuvette length, the paraxial beam approximation is no longer valid. The propagation paths of the spectral components of $\mathrm{THz}$ become not equal, becoming an additional source of errors contributing to measuring the TOF of the pulse. To minimize these errors, thin samples or short cuvettes with $l \ll Z_{R}$ should preferably be together with the $4 f$ systems. However, shorter cuvettes mean shorter optical paths and faster TOFs through a cuvette and, in consequence, lower precision of the pulse time-of-flight determination. The Rayleigh lengths can be estimated by means of Equation (A9) from Appendix A. In the $4 f$ setup from Figure 2a, large-aperture parabolic mirrors with $50.8 \mathrm{~mm}$ (2-inches) diameters and focal lengths $f_{\text {trans }}=76.2 \mathrm{~mm}$ (3-inches) and $f_{\text {focus }}=50.8 \mathrm{~mm}$ were used. Since the emitted beam waist $\omega_{\text {trans }}$ can be approximated by the radius of the Tx transmitter lens, which in our case was $\omega_{\text {trans }}=2.5 \mathrm{~mm}$, we can estimate $3 \mathrm{~mm} \leq Z_{R} \leq 175 \mathrm{~mm}$. These boundary values are representative of the spectral bandwidth of the THz pulse (Figure $2 b$ ).

The longest $Z_{R}=175 \mathrm{~mm}$ is related to the highest frequency component of the $\mathrm{THz}$ pulse, $\sim 6.0 \mathrm{THz}$, and the shortest wavelength, $\lambda \sim 0.05 \mathrm{~mm}$. It is nearly fivefold longer than the $l=5.00 \mathrm{~mm}$, the longest quartz cuvette which was used in our measurements. However, for the lowest frequency, $\sim 0.1 \mathrm{THz}$, corresponding to the longest wavelength, $\lambda \sim 3.00 \mathrm{~mm}$, $Z_{R}$ is only a fraction of $l$ and $Z_{R} / l \sim 0.6$. This pushes the paraxial beam approximation to the limit. One cannot a priori neglect additional errors related to the unequal optical paths 
of the spectral components of the pulse. For the two shorter quartz cuvettes $(l=1.00$ and $2.00 \mathrm{~mm}$ ), the Rayleigh lengths are at least $1.5 x$ longer than $l$. Therefore, one should not expect the measured TOFs to be significantly biased by non-paraxial beam propagation.

The second of the optical setups, the $2 f$ quasi-optical setup (Figure $1 \mathrm{~b}$ ), exploited a different approach to the issue of unequal optical paths. Here, the cuvette with gasoline samples was exposed to a collimated $\mathrm{THz}$ beam, occupying a midpoint between the two off-axis parabolic mirrors. The cuvette was situated at the Fourier plane, shifted by one focal length distance from the coupling mirror (first mirror on the left-hand side in Figure 1b). Smaller diameter mirrors, $25.4 \mathrm{~mm}$ (1-inch), with equal focal lengths, $f_{\text {trans }}=50.8 \mathrm{~mm}$, were used in the $2 f$ configuration.

The Rayleigh lengths of the spectral components of the $\mathrm{THz}$ pulse were estimated using the method described in the second part of Appendix A. They differ at most by only $\sim 1 \mathrm{~mm}$ for the whole bandwidth of the pulse, i.e., form 0.1-6.0 THz. More precisely, $Z_{R}=400 \mathrm{~mm}$ for the frequency of $0.1 \mathrm{THz}$ and $Z_{R}=399 \mathrm{~mm}$ for the frequency of $6.0 \mathrm{THz}$. This is not surprising since, from a geometrical optics point of view, the $2 f$ system forms a bunch of ideally parallel rays between the two mirrors.

The $2 f$ quasi-optical setup is best suited for sensitive TOF measurements when the optical path in the cuvette is long. Small changes in the refractive index can be detected in this way, which, in turn, assures extremely low detection limits for the TDS time-of-flight method. The main limitations of this configuration are unequal beam diameters of the spectral components of the pulse; this means that only samples that are uniform over a large area can be investigated using the $2 f$ setups. Gasoline solutions fulfill this requirement to a large extent, providing the saturation pressure of volatile fuel components inside a closed cuvette has been attained. This explains why the $2 f$ optical arrangement could be used together with the longest quartz cuvette as well as the TPX cuvette providing an optical path of $l=10.00 \mathrm{~mm}$.

As schematically indicated in Figure $2 b$, the size of the $\mathrm{THz}$ beam was intentionally larger than the transversal size of the cuvette. In this way, the part of the beam that passed freely through the air provided the "zero delay" time reference for pulses transmitted through gasoline samples. Any inaccuracy of the delay line position readout or temporal drift of a laser pulse could be corrected in this way. The THz waveforms were brought to the common starting point after their registration and before data analysis so that unbiased time of flights resulting exclusively from the compositional change of a mixture change could be measured.

Figure 3a depicts a digital camera photograph of the $2 f$ setup. The inset of it shows the measurement cuvette photographed against an opened diaphragm of a diameter corresponding to the diameter of the $\mathrm{THz}$ beam. Below the photograph (Figure 3b), the waveforms of the pulses measured with the TDS spectrometer without (upper graph) and with (lower graph) the cuvette are shown. Note that by placing the cuvette, the amplitude of the reference pulse drops down while the peak amplitude position in time stays the same.

\subsection{THz-TDS Time-of-Flight Method}

As shown in Figure 2, a THz pulse propagating in a medium is a superposition of plane harmonic waves with frequencies defined by its spectrum. Mathematically, the spectrum of the pulse together with the phase of each spectral component can be calculated by means of the Fourier transform [25]. 


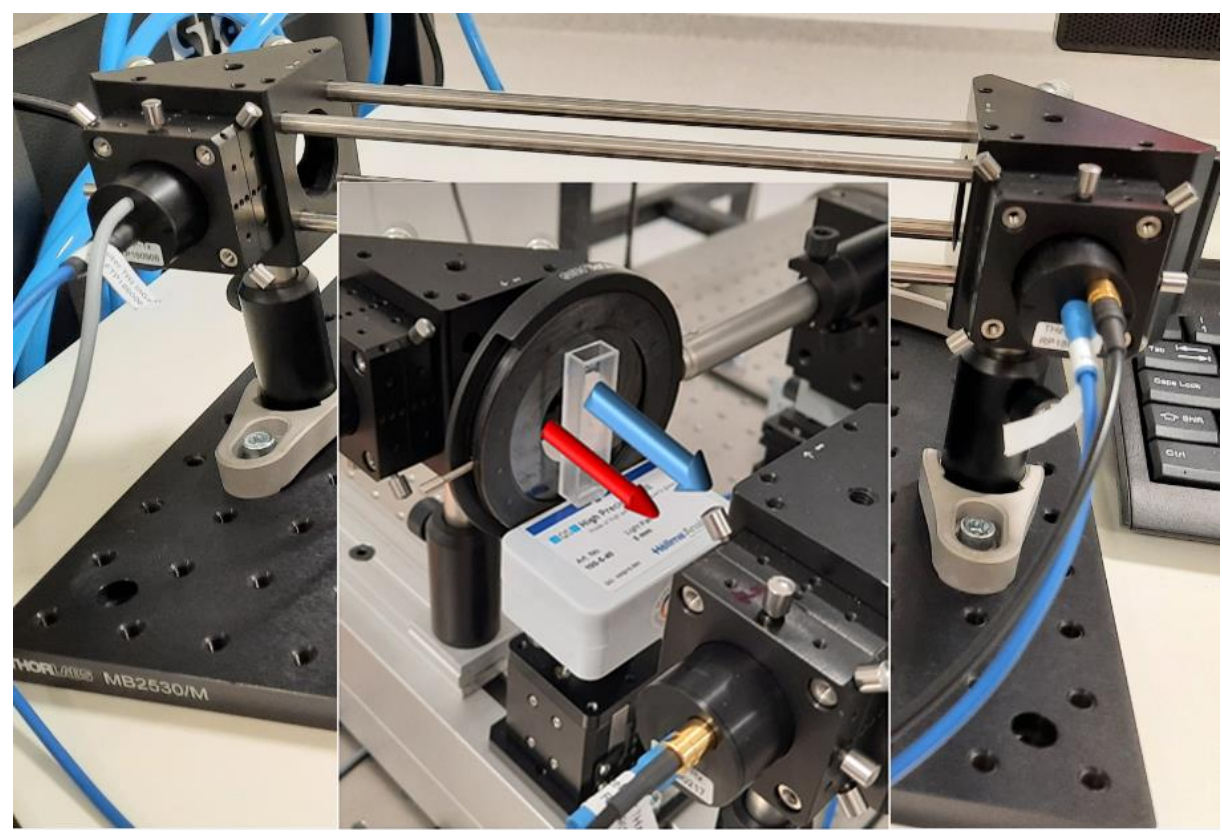

(a)

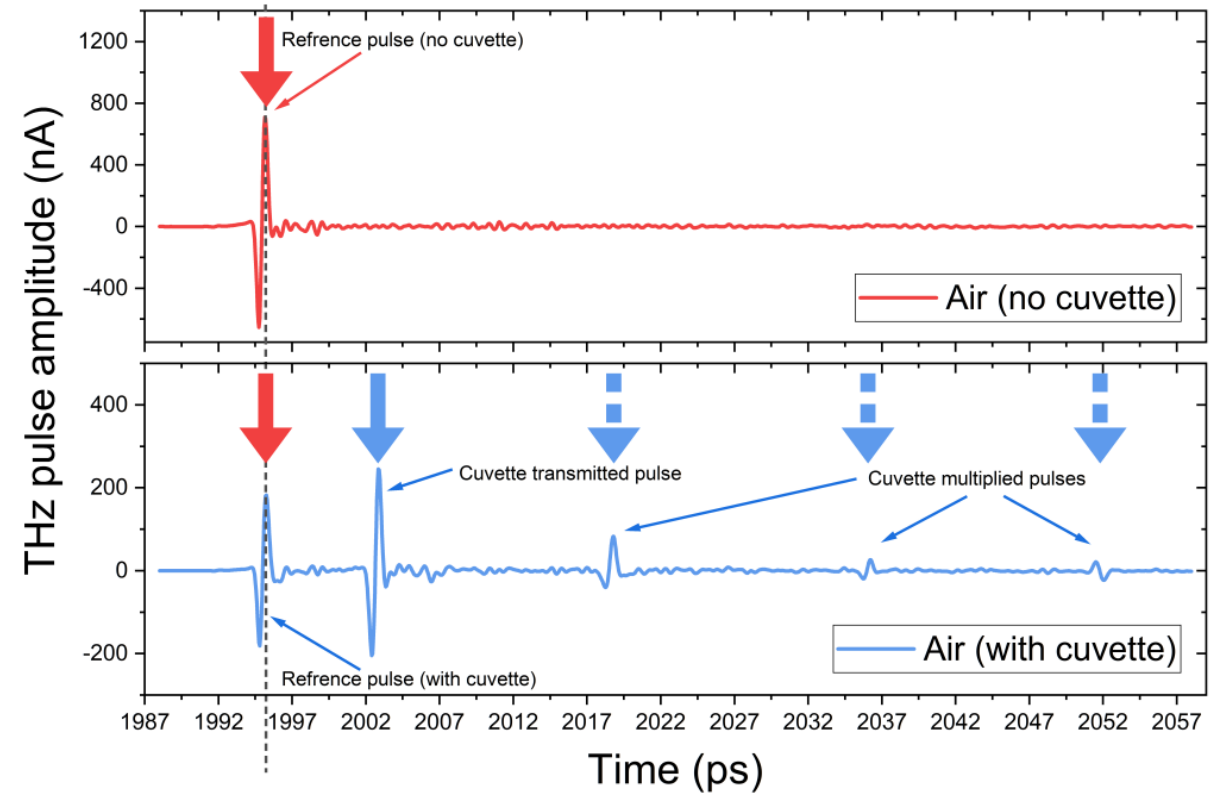

(b)

Figure 3. The reference pulse measurement principle using the $2 f$ quasi-optical setup: (a) digital camera photograph of the setup with the red arrow indicating the part of the beam where the $\mathrm{THz}$ pulse passes freely between the emitter and detector and the blue arrow indicating the beam transmitted through the cuvette; (b) TDS waveforms measured without (upper graph) and with (lower graph) the measuring cuvette. Above the pulses, the colorful arrows designate the freely passing and cuvette-transmitted parts of the beam, respectively. The pulses undergoing multiple reflections are indicated by dashed blue lines. The color code is consistent between the upper and lower graphs.

The time of flight of the pulse throughout a cuvette can be conveniently defined by a group velocity represented by the speed of the center of the mass of a wave packet. In the first and simplest approximation of negligible absorption and dispersion, the group velocity of a wave packet or pulse can be approximated by the phase velocity of its middle 
frequency [26]. A simple linear equation relates the TOF of the THz pulse in air, $t_{\text {air }}$, with the refractive index, $n_{\text {air }}$, and the length of the cuvette, $l$ :

$$
t_{\text {air }}=\frac{l}{c} n_{\text {air }}
$$

Here, $c$ denotes the speed of light in vacuum. Analogically, the TOF of the pulse, $t$, sent through a cuvette filled with a substance of the refractive index, $n$, can be calculated as:

$$
t=\frac{l}{c} n
$$

so that the difference between both times, $t-t_{\text {air }}=\Delta t$ :

$$
\Delta t=\frac{l}{c}(n-1),
$$

provides the means to determine the refractive index of the substance. Note that in Equation (3), the approximation of $n_{\text {air }} \cong 1$ was used. The accuracy of the determination of $n$ depends on the accuracy of the measured $\Delta t$. A simple calculation shows that for the cuvette length $l=10.00 \mathrm{~mm}$, corresponding to the longest of the cuvettes that were used, $\Delta(n-1) \sim 10^{-5}$ if $\Delta t \sim 10^{-15} \mathrm{~s}$. This remarkable result proves that, in principle, very low concentrations of admixtures introducing $\sim 10^{-5}$ changes in the refractive index can be detected by a straightforward THz-TDS TOF measurement. A prerequisite for such measurement is a stable position and shape of a THz pulse generated by a TDS spectrometer. In Section 3.1, we show that such accuracy was achieved with our measurement setup.

For the purpose of admixture detection in gasoline, it is more convenient to reference the time difference, $\Delta t$, to the TOF of the pulse through a cuvette filled with pure gasoline. This situation is schematically depicted in Figure $4 \mathrm{~b}$. According to Equation (3) the first of the TOFs, $t_{n D}$, is related to the refractive index of a mixture, $n_{D}$. The second TOF, the reference time-of-flight, $t_{n G}$, is related with the refractive index of gasoline and the difference between the times of flight, $\Delta t$, is calculated as:

$$
\Delta t=t_{n_{D}}-t_{n_{G}}=\frac{l}{c}\left(n_{D}-1\right)-\frac{l}{c}\left(n_{G}-1\right)=\frac{l}{c}\left(n_{D}-n_{G}\right) .
$$

The equation above can be used to plot the measured $\Delta t$ as a function of admixture concentration, providing that the model of the refractive index of the solution, $n_{D}$, is known. The refractive index of pure gasoline, $n_{G}$, can be determined, e.g., by performing the reference TOF measurement in the empty cuvette and then using Equation (3).

\subsection{Theoretical Model of the Refractive Index of Gasoline Admixture Solutions}

Since the composition of a mixture typically includes constituents belonging to different classes of compounds, a model suitable to adequately describe its refractive index must take into account a specific type of molecular interactions and specific mixing rules between the constituents. For example, it is known that a molar volume, $V$, of an idealized binary solution should follow the linear relation involving molar fractions of both elements, $\phi_{1,2}$, their molar masses, $m_{1,2}$, and molar densities, $\rho_{1,2}$, [27]:

$$
V=\frac{m_{1}}{\rho_{1}} \phi_{1}+\frac{m_{2}}{\rho_{2}} \phi_{2}
$$




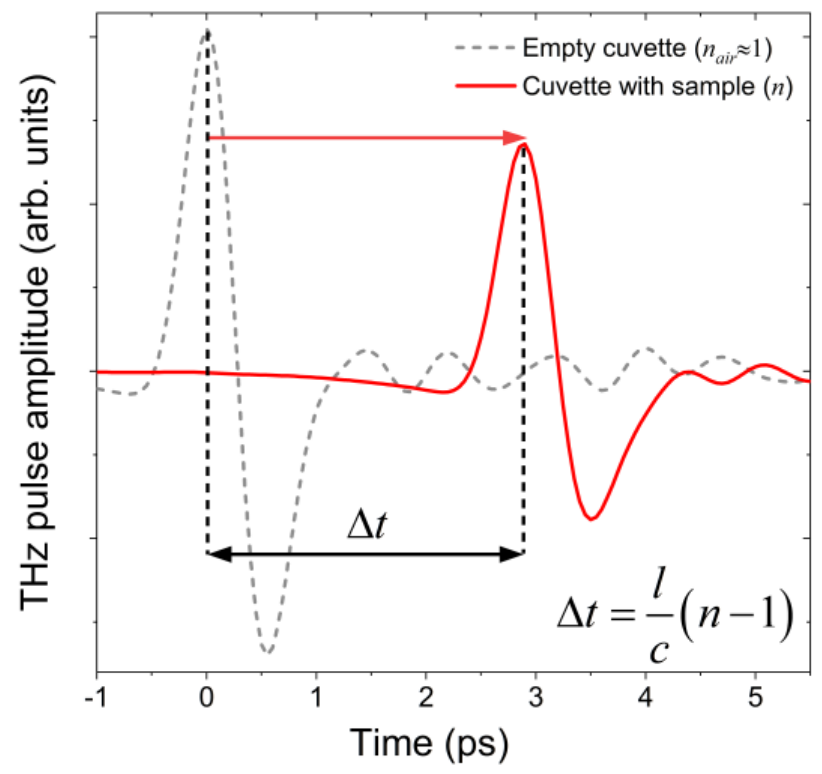

(a)

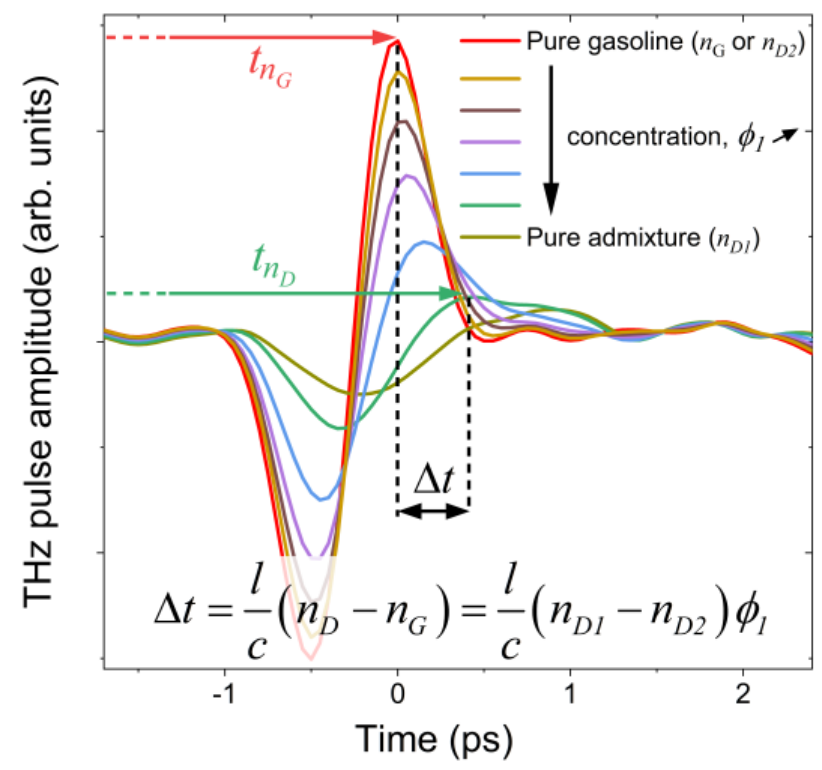

(b)

Figure 4. Graphical representation of the $\Delta t$ parameter determination: (a) for samples containing pure solvent and pure admixture when the reference pulse delay is measured with respect to an empty cuvette described by Equation (3); (b) for samples containing increasing concentration of admixture in solution as described by Equation (11).

Numerous other mixing rules can be found in the literature $[28,29]$. For example, the Lorentz-Lorenz mixing rule, given by Equation (7), is known to be particularly suitable for the description of binary solutions of organic solvents $[27,30,31]$.

The refractive index of 95-octane gasoline pseudo-binary mixtures with isopropanol has recently been studied in [32,33]. Four different mixing rules, described by four equations, namely, the Eykman (Equation (6)), Lorentz-Lorenz (Equation (7)), Newton (Equation (8)) and Gladstone-Dale (Equation (9)):

$$
\begin{gathered}
\frac{n_{D}^{2}-1}{n_{D}^{2}+0.4}=\phi_{1}\left(\frac{n_{D 1}^{2}-1}{n_{D 1}^{2}+0.4}\right)+\phi_{2}\left(\frac{n_{D 2}^{2}-1}{n_{D 2}^{2}+0.4}\right), \\
\frac{n_{D}^{2}-1}{n_{D}^{2}+2}=\phi_{1}\left(\frac{n_{D 1}^{2}-1}{n_{D 1}^{2}+2}\right)+\phi_{2}\left(\frac{n_{D 2}^{2}-1}{n_{D 2}^{2}+2}\right), \\
n_{D}^{2}-1=\phi_{1}\left(n_{D 1}^{2}-1\right)+\phi_{2}\left(n_{D 2}^{2}-1\right), \\
n_{D}-1=\phi_{1}\left(n_{D 1}-1\right)+\phi_{2}\left(n_{D 2}-1\right) .
\end{gathered}
$$

Were tested in the quoted above publications in the temperature range from $293.15 \mathrm{~K}$ to $313.5 \mathrm{~K}$. In Equations (6)-(9), the symbols $n_{D 1}$ and $n_{D 2}$ stand for the refractive index of isopropanol-gasoline mixture components $\phi_{1}$ and $\phi_{2}$, respectively. Using these equations, the refractive index of isopropanol-gasoline mixtures was found to increase linearly from $n_{D 1}$ to $n_{D 2}$, with $n_{D 2}$ belonging to the constituent of the larger absolute value of $n$ [33]. The best correspondence was found for the Eykman and Lorentz-Lorenz model; however, the differences between the measured and calculated $n_{D}$ using the Gladstone-Dale equation (Equation (9)) were also reasonably small with the absolute values $\Delta n_{D}=\left|n_{D, \exp }-\left(\phi_{1} n_{D 1}-\phi_{2} n_{D 2}\right)\right|$ not larger than $\sim 0.0016$. They applied to the situation when the gasoline and isopropanol concentrations were approximately equal $\left(\phi_{1,2} \approx 0.5\right)$. With smaller solvent or admixture concentrations, the differences were even 
smaller, approaching $\Delta n_{D} \cong 0$. The mixing rules were tested for the visible light of the wavelength $\lambda=589.3 \mathrm{~nm}$ (sodium $\mathrm{D}$ line).

In order to verify if the Gladstone-Dale mixing rule can be useful for modeling the measured pulse delays in the THz spectral range, we have employed Equation (9) using the mass fractions of isopropanol and gasoline, $\phi_{1}$ and $\phi_{2}$, respectively. With this new representation, the refractive index of the solution, $n_{D}$, is described by the relation:

$$
n_{D}=\phi_{1} n_{D 1}+\phi_{2} n_{D 2}
$$

Equation (10) defines the refractive index of a mixture as the weighted sum of the refractive indices of solvent and admixture with the weights $\phi_{1,2}$, given as the mass fractions (\%wt.) with respect to the total mass of the solution. By combining Equations (4) and (10), $\Delta t$ can be calculated as:

$$
\Delta t=t_{n_{D}}-t_{n_{G}}=\frac{l}{c}\left(n_{D 1}-n_{D 2}\right) \phi_{1} .
$$

The parameters $n_{D 1}$ and $n_{D 2}$ denote the refractive index of pure isopropanol and gasoline, respectively. Additionally, the relationship $\phi_{1}+\phi_{2}=1$ was used to derive the final form of Equation (11). Details of the derivation can be found in Appendix B.

The Gladstone-Dale mixing rule predicts a linear relationship between $\Delta t$ and the admixture concentration $\phi_{1}$.

\section{Results}

\subsection{Time Stability Performance of the THz-TDS Setup}

The stability performance of the THz-TDS spectrometer was verified with the beam delivery part and photoconductive antennas tightly closed in a plexiglass container, from which ambient air was removed by purging the container with purified clean dry air (CDA). The TOFs of the pulses were measured in a collimated beam geometry, i.e., with the $2 f$ quasi-optical setup. At first, the waveforms of the THz pulses were measured in CDA. Then, the container air was mixed with a small amount of humid ambient air, and the registration of the waveforms was repeated. In both cases, the THz pulses were propagated along the same optical paths with and without the quartz cuvette.

The differences between the waveforms registered in both types of air are shown in Figure 5. The figure compares the average waveforms obtained by subsequent acquisition and averaging of 1,000 THz pulses. The dry and moist air time profiles are almost identical, and the differences between them can only be observed on a sub-ps time scale.

The fitted peak amplitudes of the pulses are shown by the graphs from the lower row of the figure. They have small fit errors of 1 and $2 \mathrm{fs}$, respectively, for the reference and the cuvette transmitted pulse. One can be sure that the time-shift between the pulses was not caused by numerical artifact but resulted from moisture in the plexiglass box. Such a conclusion is confirmed by a flawless systematical trend of the measured data points before and after the maximum of the pulse, as indicated by the insets of Figure $5 c$,d.

Apparently, the shift of the reference pulse in time corresponds inevitably to a similar shift of the quartz cuvette transmitted pulse. Note that in both cases, the measured time-offlight difference was the same: $\sim 1$ fs. Somewhat larger fit errors for the cuvette transmitted pulses are likely caused by dispersion introduced by the input and output cuvette walls made of quartz glass. This confirms that the strategy of using an unobscured part of the beam as the reference is generally correct and that pulse instabilities caused by the TDS spectrometer can be compensated with up to an fs precision.

To test the time stability performance of the TDS spectrometer, we have continuously observed the peak amplitude position of the $\mathrm{THz}$ pulse over a $\sim 2.5 \mathrm{~h}$ time (Figure 6 ). The system was running freely, and the waveforms of the pulses were registered in more or less equal time intervals of $\sim 12 \mathrm{~min}$. Over the whole period, the pulse shifted by maximally $31 \mathrm{fs}$ towards shorter times and stabilized the position approx. $50 \mathrm{~min}$ after the spectrometer was turned on. Then, small-scale fluctuations with less than $1 \mathrm{fs}$ deviation were observed over 
the next $\sim 45 \mathrm{~min}$. During the last hour of observations, the pulsed drifted again towards its initial position by $\sim 9 \mathrm{fs}$.

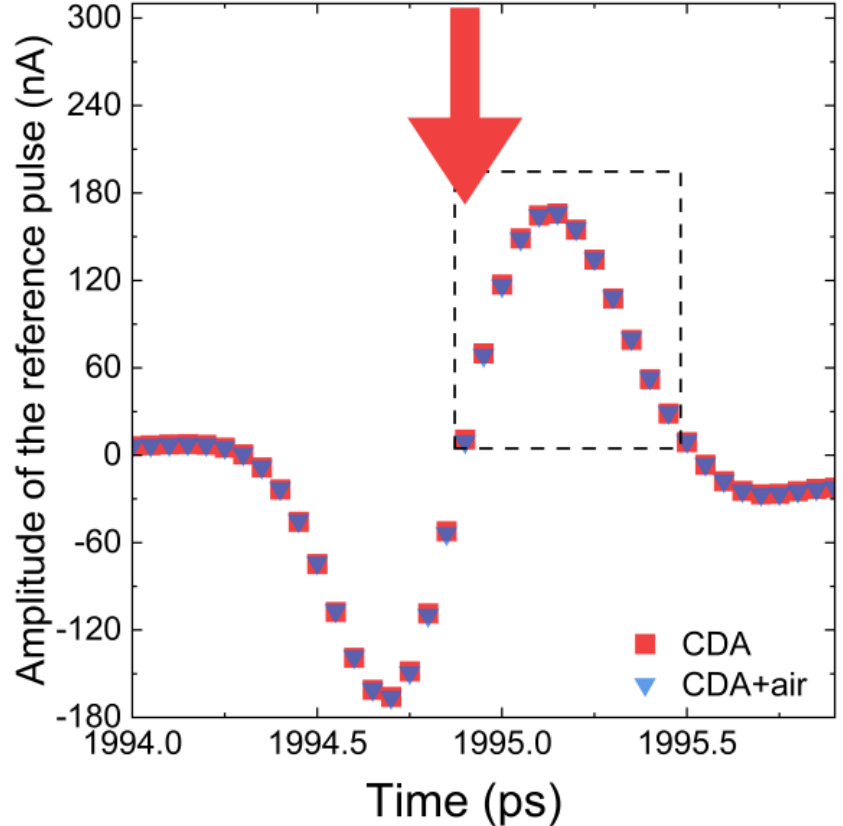

(a)

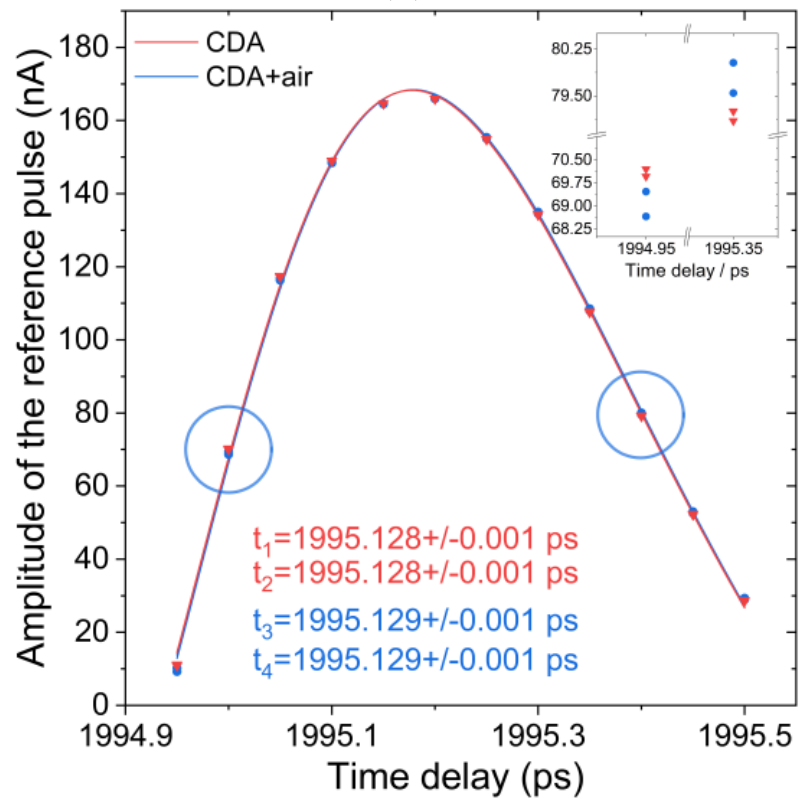

(c)

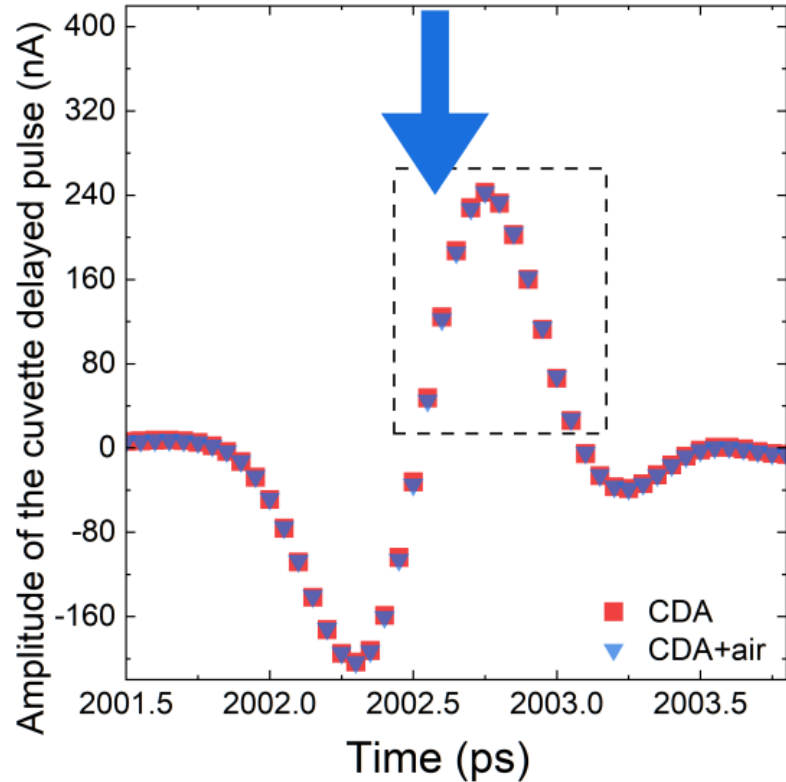

(b)

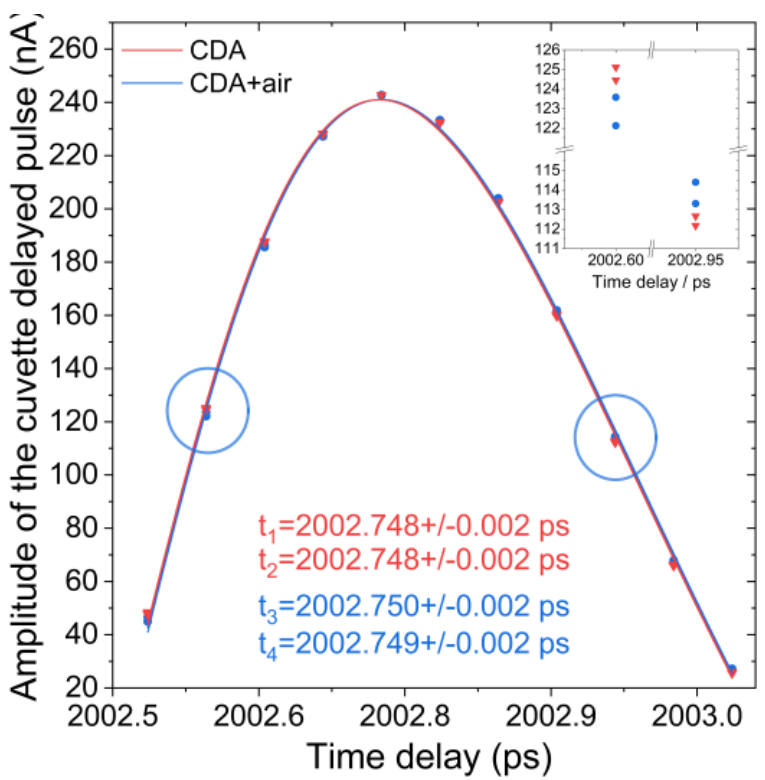

(d)

Figure 5. Time performance of the THz-TDS system: (a) time profiles of the reference pulses registered in CDA and moist air; (b) time profiles of the pulses transmitted through $5.00 \mathrm{~mm}$ long quartz cuvette. The color code of the arrows indicated above the pulses is the same as in Figure 3. (c,d) Close-up view of the peak amplitude regions showing actually measured points and their numerical fits with model peak functions. The insets of the figures present, on a smaller scale, the data marked by blue circles. The numbers below the curves indicate the fitted positions of the peak amplitudes together with the fit errors. Note that the time positions of the pulses can be determined with 1 or 2 fs accuracy, respectively, for the reference and cuvette transmitted pulses.

A special procedure was applied to correct underperformances of the TDS system related to pulse position instabilities. The registered waveforms were always brought 
to the common "zero time delay" point before data analysis in the procedure, which is schematically depicted in Figure $6 \mathrm{a}, \mathrm{b}$. For each of the waveforms, the peak amplitude position of the reference pulse was determined by a numerical fit. Its numerical value was next subtracted from the time data, setting the reference "zero time" point for all the waveforms in the measurement series (Figure 6b). Since we have checked that the reference and cuvette transmitted pulses always shift simultaneously, we can consider that any pulse delays measured with the cuvettes filled with gasoline samples are likely caused by admixture concentration change and not the drift of the pulse itself.

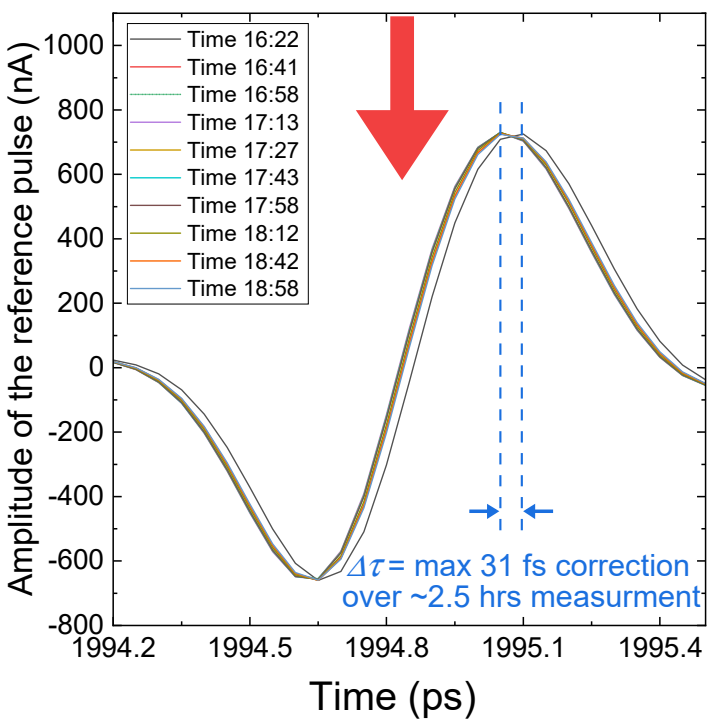

(a)

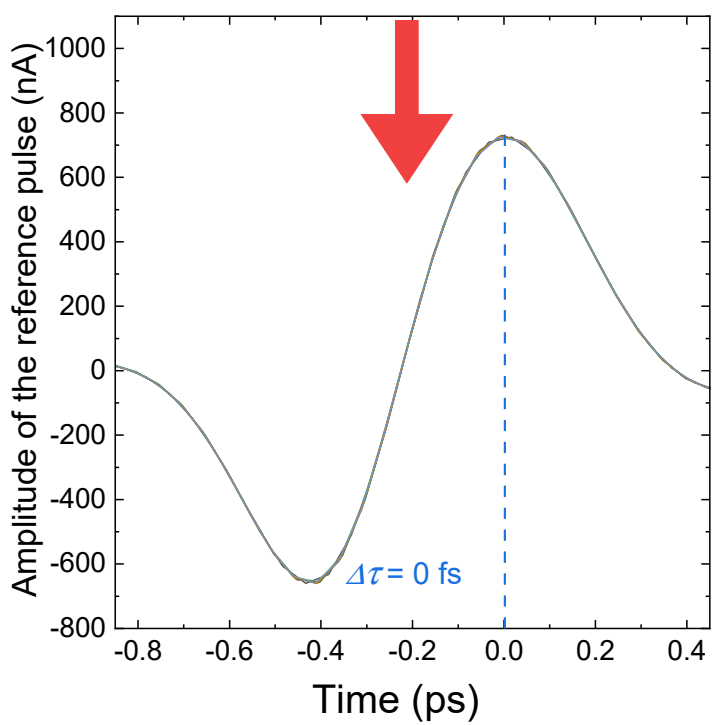

(b)

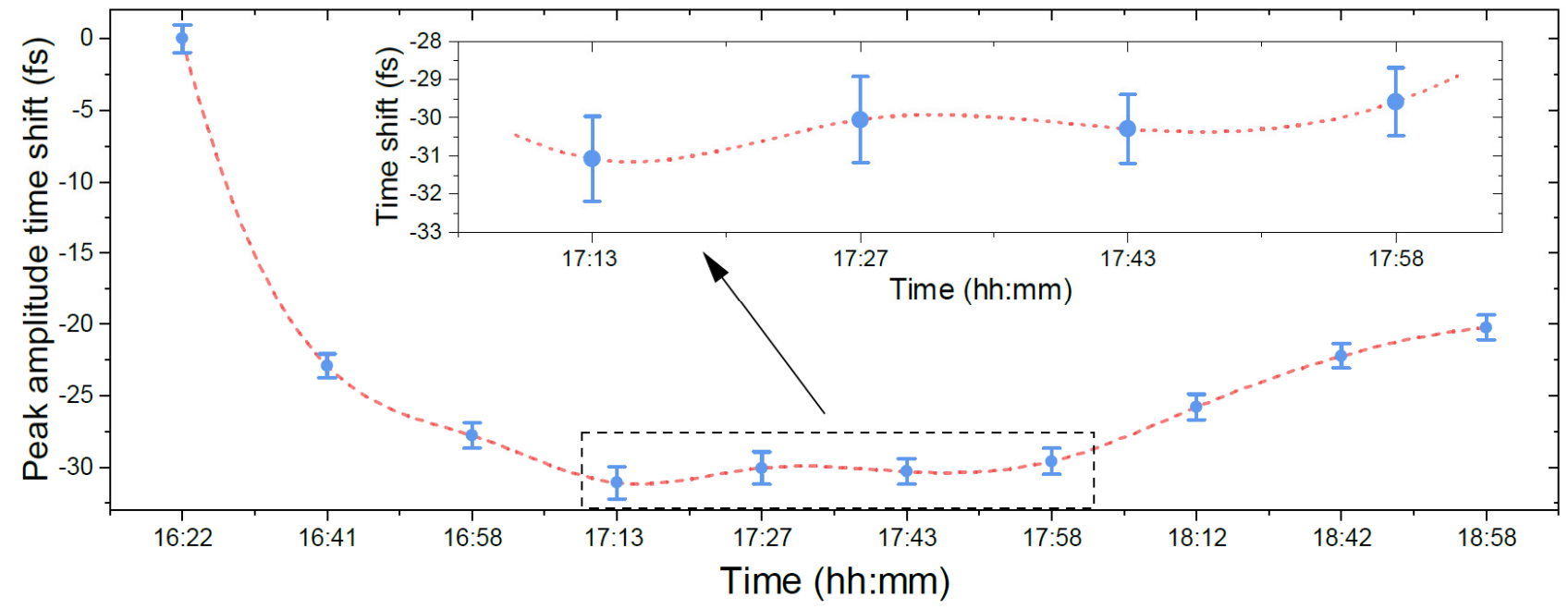

(c)

Figure 6. Time correction procedure of the THz pulse: (a) Actually measured waveforms of the reference pulse before correction; (b) The same waveforms brought to the common "zero delay" time after correction. The applied correction is also valid for the pulses propagating through gasoline samples during the measurements. The color code of the arrows above the pulses is used consistently with previous figures; (c) The measured evolution of the peak amplitude of the THz reference pulse during approx. $2.5 \mathrm{~h}$-long series of the measurements. The positions of the peaks with fit errors were determined by numerical fit with a predefined function. The inset of the graph shows small-scale oscillations of the pulse position in detail.

While the absolute peak amplitude positions of the reference pulses could be determined in CDA with an accuracy of 1-2 fs, the instabilities of the $\mathrm{THz}$ pulse position 
measured with gasoline samples (or ambient air) were higher, $10-100 \mathrm{fs}$. They were strongly dependent on admixture type, length of the cuvette and absorbance properties resulting from the admixture concentration in the solution. These instabilities were reasonably smaller than typically measured pulse TOFs and were most likely caused by inhomogeneity and density fluctuations of the liquids.

\subsection{Determination of the Refractive Indices of Pure Isopropanol and Pure Gasoline}

In order to quantitatively validate the THz-TDS TOF method, we completed a series of test measurements with pure gasoline and pure isopropanol used as the samples. The TOFs of pulses transmitted through all three quartz cuvettes of the lengths $l=1.00,2.00$ and $5.00 \mathrm{~mm}$ were measured. The pulse positions differences, $\Delta t$, were next calculated with respect to the positions of the pulses transmitted through empty cuvettes (reference pulses), as indicated in Figure 7.

Table 1. The TOFs and refractive indices of pure isopropanol and gasoline measured by THz-TDS.

\begin{tabular}{|c|c|c|c|c|}
\hline Cuvette Length & $\mathrm{TOF}_{\mathrm{Quartz} \text { cuvette }} \pm \delta^{1}$ (ps) & $\mathrm{TOF}_{\text {liquid }} \pm \delta$ (ps) & $\Delta t \pm \delta(\mathrm{ps})$ & THz Ref. Index $n \pm \delta$ \\
\hline \multicolumn{5}{|c|}{ Isopropanol } \\
\hline$l=1.00 \mathrm{~mm}$ & $2613.09 \pm 0.05$ & $2614.78 \pm 0.05$ & $1.69 \pm 0.10$ & $1.507 \pm 0.030$ \\
\hline$l=2.00 \mathrm{~mm}$ & $2612.93 \pm 0.05$ & $2616.30 \pm 0.07$ & $3.37 \pm 0.12$ & $1.506 \pm 0.020$ \\
\hline \multirow[t]{2}{*}{$l=5.00 \mathrm{~mm}$} & $2612.99 \pm 0.05$ & $2621.45 \pm 0.10$ & $8.46 \pm 0.15$ & $1.5076 \pm 0.0090$ \\
\hline & & & & $n_{\text {iso }}=<1.50687 \pm 0.00081>^{2}$ \\
\hline \multicolumn{5}{|c|}{ Gasoline } \\
\hline$l=1.00 \mathrm{~mm}$ & $2613.07 \pm 0.05$ & $2614.51 \pm 0.06$ & $1.44 \pm 0.11$ & $1.432 \pm 0.033$ \\
\hline$l=2.00 \mathrm{~mm}$ & $2612.94 \pm 0.05$ & $2615.80 \pm 0.08$ & $2.86 \pm 0.13$ & $1.429 \pm 0.020$ \\
\hline \multirow[t]{2}{*}{$l=5.00 \mathrm{~mm}$} & $2612.98 \pm 0.05$ & $2620.17 \pm 0.09$ & $7.19 \pm 0.14$ & $1.4314 \pm 0.0084$ \\
\hline & & & & $n_{g a s}=<1.4308 \pm 0.0016>$ \\
\hline
\end{tabular}

${ }^{1} \delta$ Fit errors. ${ }^{2}<\ldots>$ Average values of the refractive indices with standard deviations.

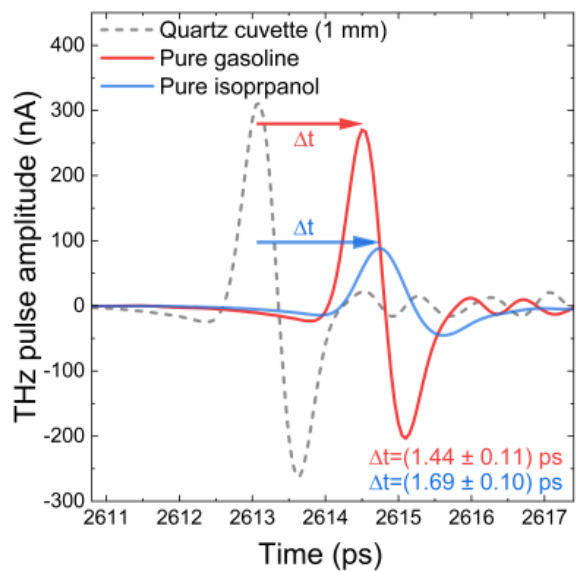

(a)

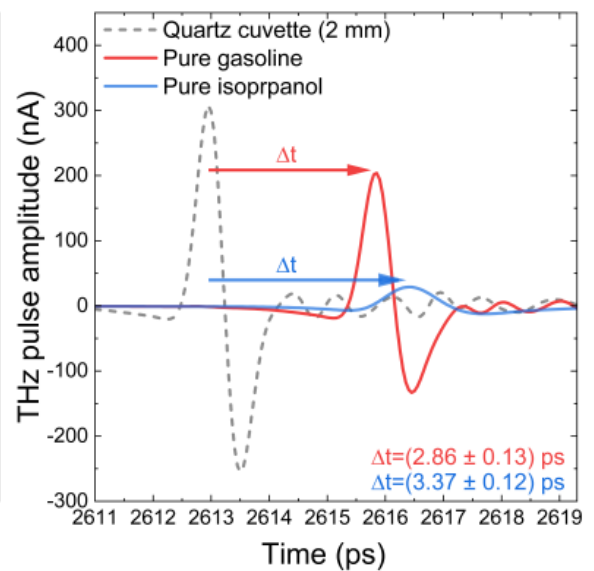

(b)

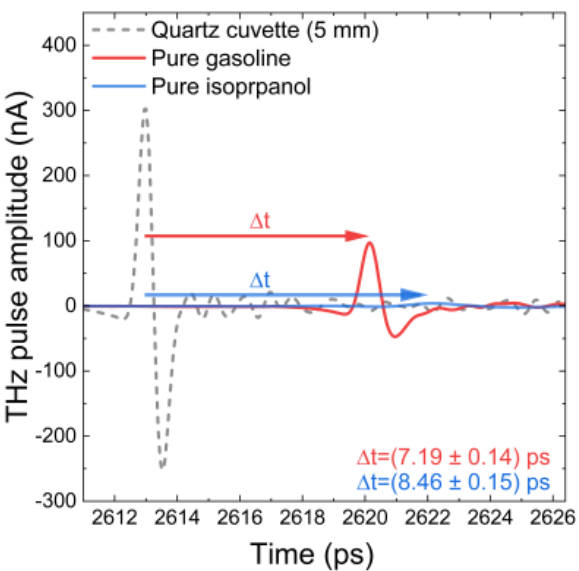

(c)

Figure 7. Determination of the refractive index of pure isopropanol and 95-octane gasoline in: (a) quartz cuvette of length $l=1.00 \mathrm{~mm}$; (b) similar cuvette with length $l=2.00 \mathrm{~mm}$; (c) and a similar cuvette with length $l=5.00 \mathrm{~mm}$. The real measured maxima positions of the pulses are indicated in Table 1.

The measurements were conducted in ambient air with the $4 f$ system optical beam geometry, and no time correction of the peak amplitude positions was applied before data analysis. This was largely justified since no substantial pulse drift was expected in a single minute duration data acquisition time. 
The registered waveforms of the THz pulses are shown in Figure 7. Each of the graphs represents measurements belonging to different cuvette lengths. The empty cuvette (reference)-, gasoline- and isopropanol-transmitted pulses are indicated by different colors of the lines. Additionally, the dashed line was used for individual marking of the reference pulses.

The amplitudes of the pulses decrease with the increasing cuvette lengths. Since gasoline has a weak absorption coefficient, resulting mostly from transient dipole moments induced by collisions [34], this decrease is much faster for isopropanol, which is a strongly polar molecule with a permanent dipole moment and a large $\mathrm{THz}$ radiation absorption coefficient $[35,36]$. A similar conclusion also applies to the measured pulse delays, which are longer for isopropanol than for gasoline. This observation is explained by the larger absolute value of the refractive index of isopropanol as compared to gasoline. Note also that for both isopropanol and gasoline, the pulse delays increase with the increasing length of the cuvette, as expected from Equation (3).

The results of numerical fits of peak amplitude positions of all pulses were summarized in Table 1. Starting from the second column, this table indicates the fitted times of flight measured with the empty cuvettes and cuvettes with isopropanol or gasoline. The third column, $\Delta t$, indicates the calculated differences of the times of flight. The refractive indices, $n$, from the last column of the table were calculated by means of the

Equation (3). The error of $n$ takes into account only the error of $\Delta t$, assuming that the other parameters from Equation (3) were known with the absolute accuracy (their errors were assumed to be zero).

The average refractive indices of isopropanol and gasoline, indicated in the table in the brackets, represent the average values calculated, taking into account all three cuvette lengths. Their numerical values were compared with the values reported in the literature by other authors [11,37-39]. The outcome of the comparison is summarized in Table 2. Generally, we can state a good agreement between these data, as the measured refractive indices $n_{\text {iso }}=<1.50687 \pm 0.00081>$ and $n_{\text {gas }}=<1.4308 \pm 0.0016>$ place themselves within boundaries reported in the publications. This confirms that the THz-TDS time-of-flight method can provide accurate and valuable data regarding the refractive index of liquids.

Table 2. Comparison between the measured and reference refractive indices of pure isopropanol and pure gasoline. The source of the references is indicated below the table.

\begin{tabular}{ccc}
\hline Substance & TOF $\boldsymbol{R}$ & Reference $\boldsymbol{n}$ \\
\hline Isopropanol & $n_{\text {iso }}=1.50687 \pm 0.00081$ & $n=1.50^{1}$ \\
Gasoline & $n_{\text {gas }}=1.4308 \pm 0.0016$ & $n=1.415-1.441^{2-4}$ \\
\hline
\end{tabular}

${ }^{1}$ Huang et al. [37], average 0.2-1.4 THz. ${ }^{2}$ Arik et al. [11], average 0.1-1.1 THz. ${ }^{3}$ Jin et al. [38], unspecified octane number, average 0.2-2 THz. ${ }^{4}$ Al-Douseri et al. [39], @1 THz, 93-octane gasoline.

Based on this conclusion, we have experimentally verified the appropriateness of our approach in the measurements of isopropanol solutions with systematically varied isopropanol concentrations.

\subsection{Investigations of THz Pulse Time of Flights in Gasoline Solutions with Isopropanol}

Figure 8 shows the uncorrected, raw data waveforms of $\mathrm{THz}$ pulses obtained after propagation in gasoline mixtures containing isopropanol alcohol. The registrations were taken in quartz cuvettes of all three lengths. The cuvettes were located in the Fourier plane of the $4 f$ quasi-optical beam delivery setup. The pulses indicated by the red arrow served as a "zero-delay" time reference for the later applied pulse position correction procedure (Section 3.1). They had to be acquired in separate registrations directly before time-of-flight measurements because the size of the focused $\mathrm{THz}$ beam in the $4 f$ system is incomparable with the transverse dimensions of the cuvette. Despite different methods of registration, the reference waveforms were used in the time correction procedure that was the same for the $4 f$ and $2 f$ systems. 
The waveforms indicated by green, red and blue lines correspond to $l=1.00,2.00$ and $5.00 \mathrm{~mm}$ cuvettes, respectively. The actual isopropanol concentrations related to each of the series are summarized in Table 3. It is necessary to understand that the chosen range of isopropanol concentration in gasoline had to be carefully adjusted to the lengths of the cuvettes because transmission of $\mathrm{THz}$ radiation could be very limited over long propagation paths in the cuvettes.

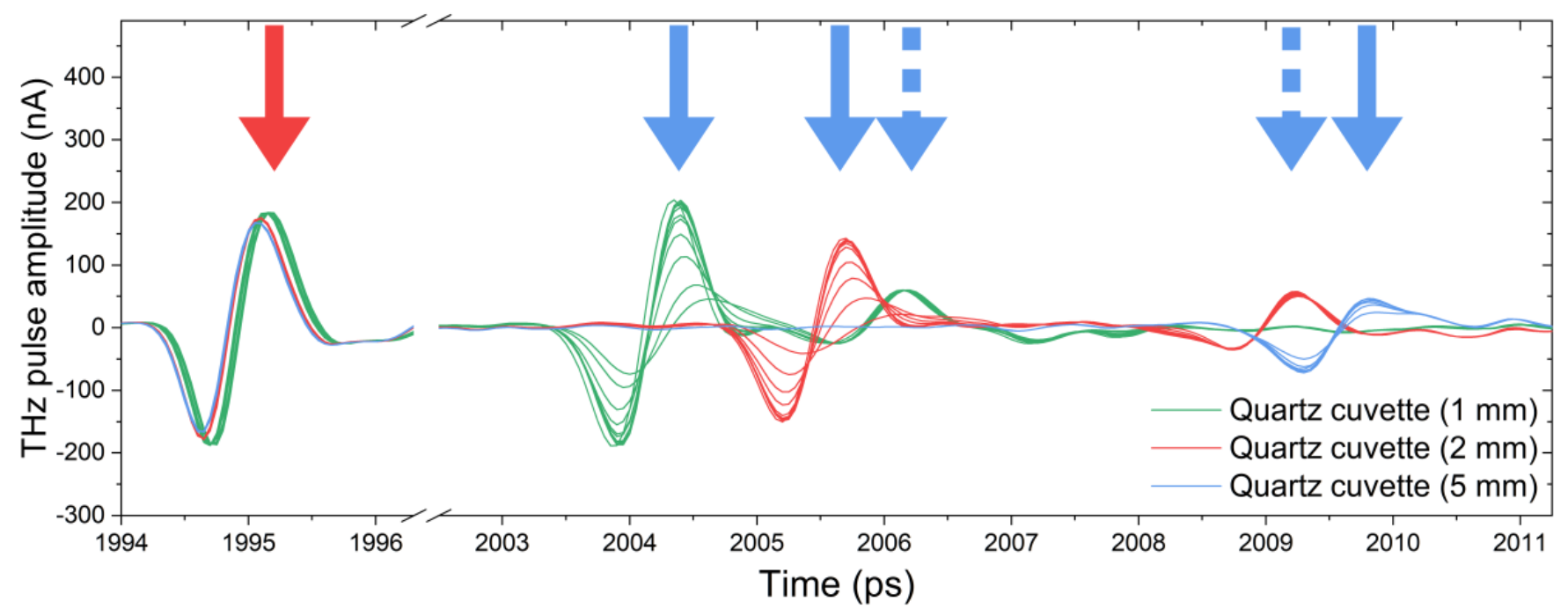

Figure 8. Waveforms of the $\mathrm{THz}$ pulses registered with $l=1.00,2.00$ and $5.00 \mathrm{~mm}$ quartz cuvettes and gasoline isopropanol admixture samples. The curves represent raw, uncorrected data as measured with the THz-TDS spectrometer and $4 f$ quasi-optical beam delivery system. The leftmost peaks belonging to the $\mathrm{THz}$ pulses passing through the air without quartz cuvettes were used to compensate for drifts and fluctuations of the pulses transmitted through gasoline samples. The color code of the arrows above the waveforms is consistent with previous figures.

The blue arrows in the figure indicate the $\mathrm{THz}$ pulses after their interaction with gasoline samples and the dashed blue arrows-the pulses multiplied by the cuvettes after internal reflections.

The measured TOFs were compared with predictions of Equation (11). The results of the comparison are depicted in Figure 9. In the first graph (Figure 9a), the measured TOF differences, $\Delta t$, were plotted against the mass fractions of isopropanol, $\phi_{1}$, from Table 1 . The "zero-delay" time difference, $\Delta t=0$, corresponds to the positions of pure gasoline pulses belonging to each of the series from Figure 8.

A common characteristic that is apparent in Figure $9 \mathrm{a}$ is that, independent of cuvette lengths, the measured $\Delta t$ increases approximately linearly with the isopropanol concentration. Moreover, for the fixed absolute value $\phi_{1}, \Delta t$ is proportional to the length of the cuvette, $l$, and is manifested by increasing slopes of the measured points. Additionally, the time separation between them along the ordinate is visibly better when the optical lengths are longer, meaning that longer cuvettes are much better suited for sensitive measurements of trace concentrations of admixture in the solution.

Even if the broadest span of the investigated mass fractions of isopropanol was from 0 to $750,000 \mathrm{ppm}$, i.e., 0 to $75 \% \mathrm{wt}$., such a large range of concentrations could only be covered in the shortest $1.00 \mathrm{~mm}$ cuvette because in other cases, the limited transmission of $\mathrm{THz}$ radiation precluded the registrations of the pulse waveforms with sufficient accuracy. 
Table 3. Concentrations and mass fractions of isopropanol in gasoline mixtures.

\begin{tabular}{|c|c|c|}
\hline Cuvette Length & $\begin{array}{c}\text { Isopropanol Concentration } \\
(\% w t .)\end{array}$ & $\begin{array}{l}\text { Isopropanol Mass } \\
\text { Fraction, } \phi_{1}(\mathrm{ppm})\end{array}$ \\
\hline \multirow{12}{*}{$l=1.00 \mathrm{~mm}$} & 0.00 & 0 \\
\hline & 0.05 & 500 \\
\hline & 0.20 & 2000 \\
\hline & 0.50 & 5000 \\
\hline & 1.00 & 10,000 \\
\hline & 2.00 & 20,000 \\
\hline & 5.00 & 50,000 \\
\hline & 6.25 & 62,500 \\
\hline & 12.5 & 125,000 \\
\hline & 25.00 & 250,000 \\
\hline & 50.00 & 500,000 \\
\hline & 75.00 & 750,000 \\
\hline \multirow{10}{*}{$l=2.00 \mathrm{~mm}$} & 0.00 & 0 \\
\hline & 0.05 & 500 \\
\hline & 0.20 & 2000 \\
\hline & 0.50 & 5000 \\
\hline & 1.00 & 10,000 \\
\hline & 2.00 & 20,000 \\
\hline & 6.25 & 62,500 \\
\hline & 12.50 & 125,000 \\
\hline & 25.00 & 250,000 \\
\hline & 50.00 & 500,000 \\
\hline \multirow{7}{*}{$l=5.00 \mathrm{~mm}^{1}$} & 0.00 & 0 \\
\hline & 0.05 & 500 \\
\hline & 0.20 & 2000 \\
\hline & 0.50 & 5000 \\
\hline & 1.00 & 10,000 \\
\hline & 2.00 & 20,000 \\
\hline & 5.00 & 50,000 \\
\hline
\end{tabular}

${ }^{1}$ Data of the 1st series only.

The calculated theoretical dependence of $\Delta t$ on the mass fraction $\phi_{1}$ is shown in Figure $9 \mathrm{~b}$. It corresponds to the relation predicted by Equation (11). It is, therefore, not surprising that an overlap between the measured and theoretical data shown in Figure $9 \mathrm{c}$ is reasonably good.

The solid lines on this and the preceding figure (Figure $9 \mathrm{~b}$ ) resemble ab initio calculations with average values of the refractive indices from Table 2 . While the correspondence seems better for $l=1.00$ and $2.00 \mathrm{~mm}$ cuvettes, there seems to be a systematic difference towards longer measured $\Delta t$ (as compared to the calculations) for the $l=5.00 \mathrm{~mm}$ cuvette. Since all of the points on this figure represent the time-corrected data, which to a large extent are free from the spectrometer pulse drift, we interpret this visible discrepancy in terms of unequal optical path lengths and longer propagation times of the low-frequency spectral components of THz pulse. As explained in Section 2.2.2, the Rayleigh lengths of the lowfrequency components of the pulse can be considerably shorter than the $5.00 \mathrm{~mm}$ optical path of the cuvette, resulting in longer propagation distances and times of flight throughout the cuvette as compared to para-axially propagating high-frequency components.

As mentioned above, for all cuvette lengths, the ab initio calculations used the same average values of the refractive indices of isopropanol $\left\langle n_{i s o}\right\rangle$ and gasoline $\left\langle n_{g a s}\right\rangle$. More precisely, the same constant value of $\Delta n$ difference, $\Delta n=n_{\text {iso }}-n_{\text {gas }}$, was used according to the functional dependence resulting from the mathematical form of Equation (11). Since these average values do not necessarily represent an optimum, we have additionally fitted the lines to the experimentally measured $\Delta t$ and calculated the optimal $\Delta n$ from the slopes of the fits. The fitted functions are shown on the Figure $9 c$ as dash-dotted lines and the 
fitted $\Delta n$ determined in this way are: $\Delta n \cong 0.0576$ for $l=1.00 \mathrm{~mm}, \Delta n \cong 0.0660$ for $l=2 \mathrm{~mm}$ and $\Delta n \cong 0.1130$ (1st run) and $\Delta n \cong 0.1452$ ( 2 nd run) for $l=5.00 \mathrm{~mm}$.

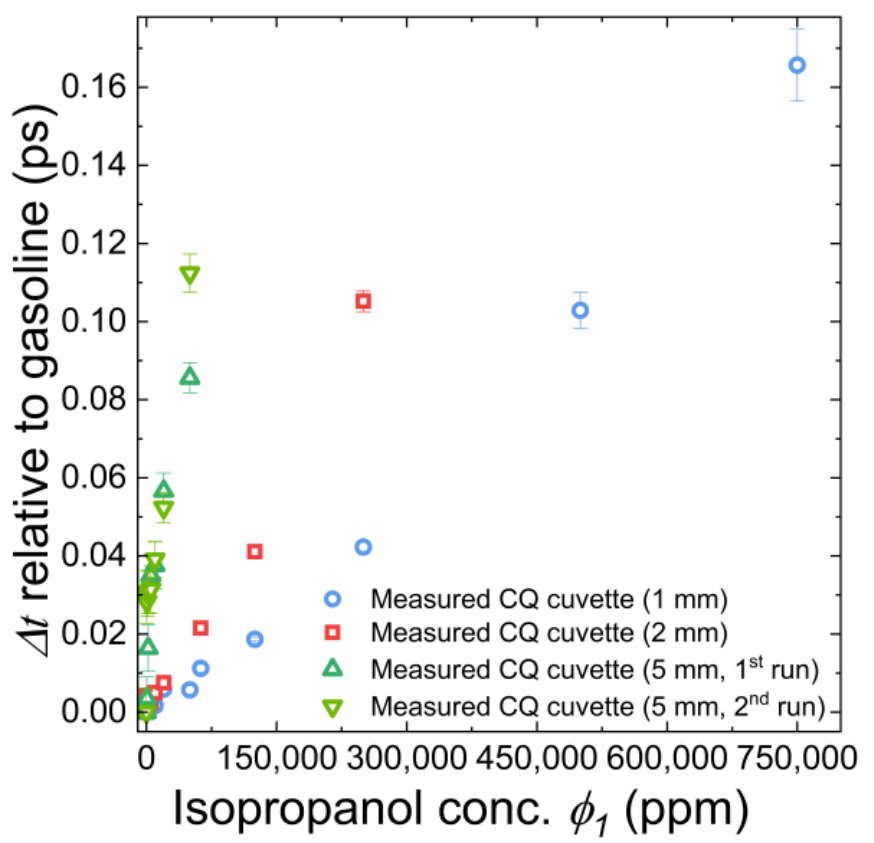

(a)

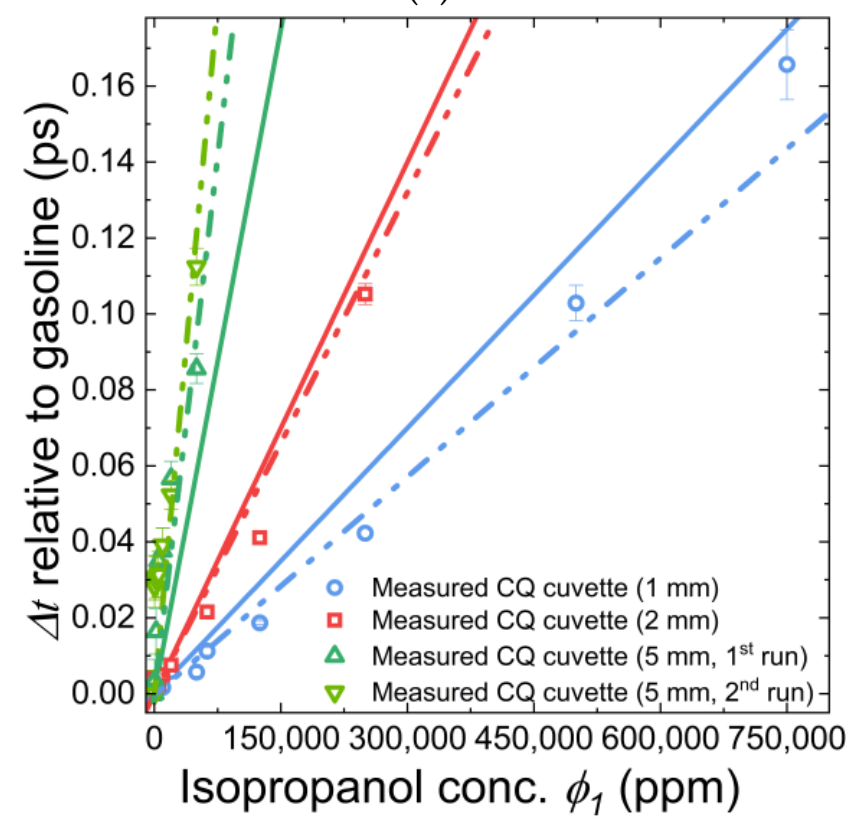

(c)

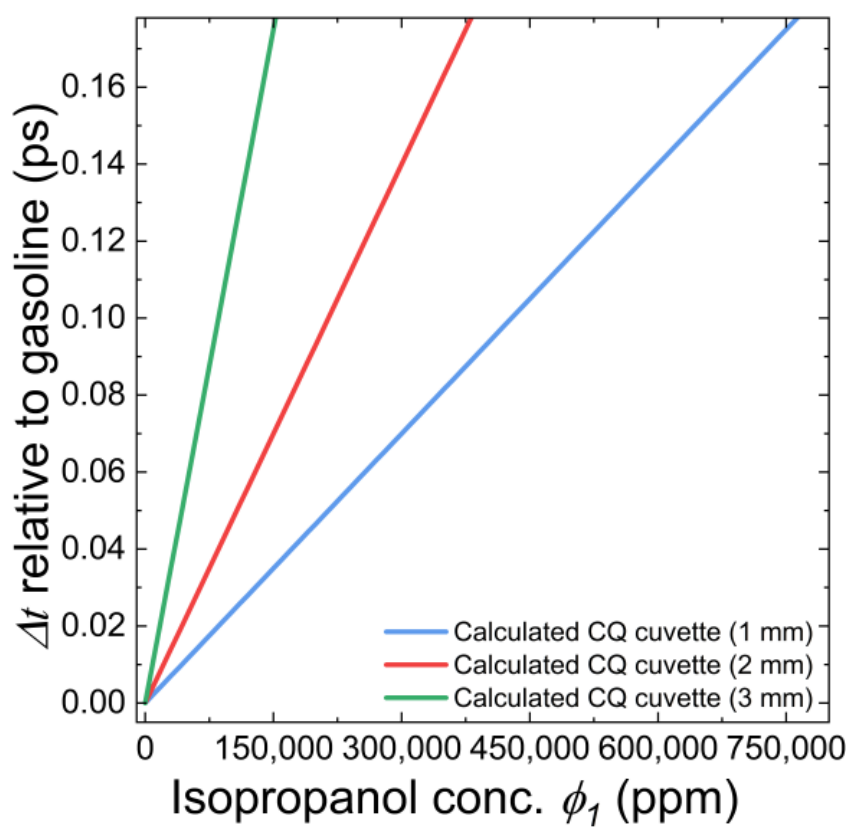

(b)

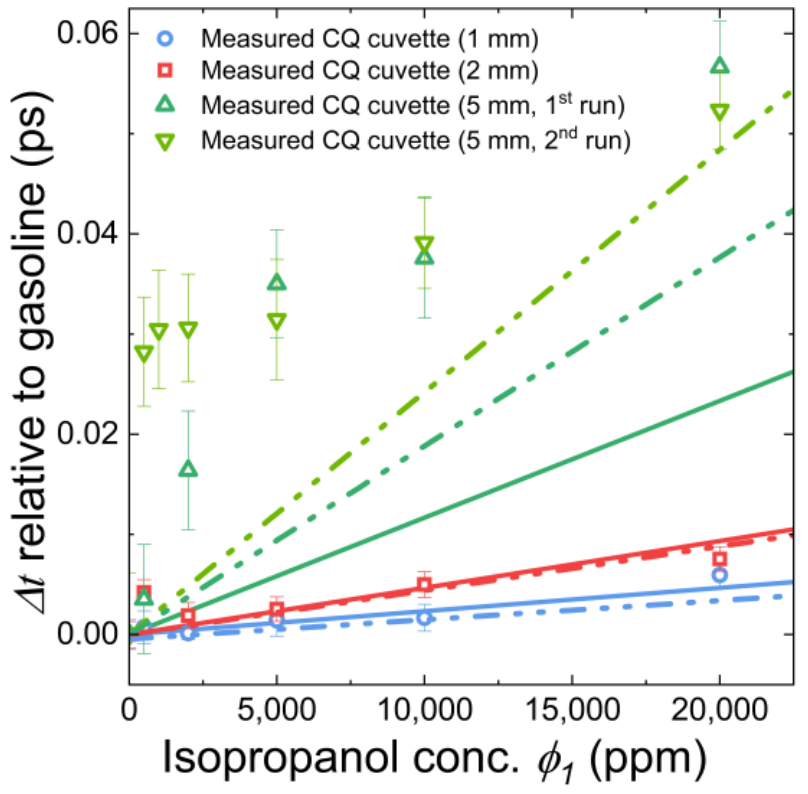

(d)

Figure 9. The measured $\mathrm{THz}$ pulse delays in gasoline containing isopropanol as a function of isopropanol concentration. (a) The results obtained with quartz cuvettes of lengths $l=1.00,2.00$ and $5.00 \mathrm{~mm}$; (b) theoretical delays calculated based on the Gladstone-Dale mixing rule given by Equation (11); (c) comparison between the measurements and calculations: the solid lines are the same as these of panel (b) and represent ab initio calculations, while the dash-dotted lines are the results of the best numerical fit; (d) magnification of data from panel (c) in the concentration range from 0 to $22,500 \mathrm{ppm}$. 


\subsection{Limits of Detection of Water and Isopropanol Admixtures in Gasoline Solutions}

The sensitivity of admixture detection was further enhanced when the quartz glass cuvettes were replaced by the TPX cuvette offering the longest optical paths of $l=10.00 \mathrm{~mm}$. We have verified in a separate measurement that despite its thicker $5 \mathrm{~mm}$ walls, as compared to the $1.25 \mathrm{~mm}$ walls of the quartz cuvettes (Section 2.1), the TPX cuvette was characterized by a much lower transmission loss of $\sim 1-5 \mathrm{~dB}$ over the whole pulse bandwidth. The corresponding transmission loss of quartz cuvettes was $\sim 14-45 \mathrm{~dB}$. The $2 f$ quasi-optical beam delivery setup was chosen because, only in this case, the Rayleigh lengths of all spectral components of the $\mathrm{THz}$ pulse could be sufficiently longer than the propagation lengths in the cuvette. The new set of gasoline samples containing only small concentrations of admixtures was prepared. The corresponding mass fractions of isopropanol and water were $0-2000 \mathrm{ppm}$ and $0-3000 \mathrm{ppm}$, respectively. The results obtained in this experimental configuration are shown in Figure 10.

In agreement with previous observations, the measured pulse delays exhibited a linear dependence on both isopropanol and water mass fractions, $\phi_{1}$. This is confirmed by numerical line fitting to the measured data points represented in Figure 10 by dash-dotted lines. The main difference was the agreement with the model of the refractive index of the mixture.

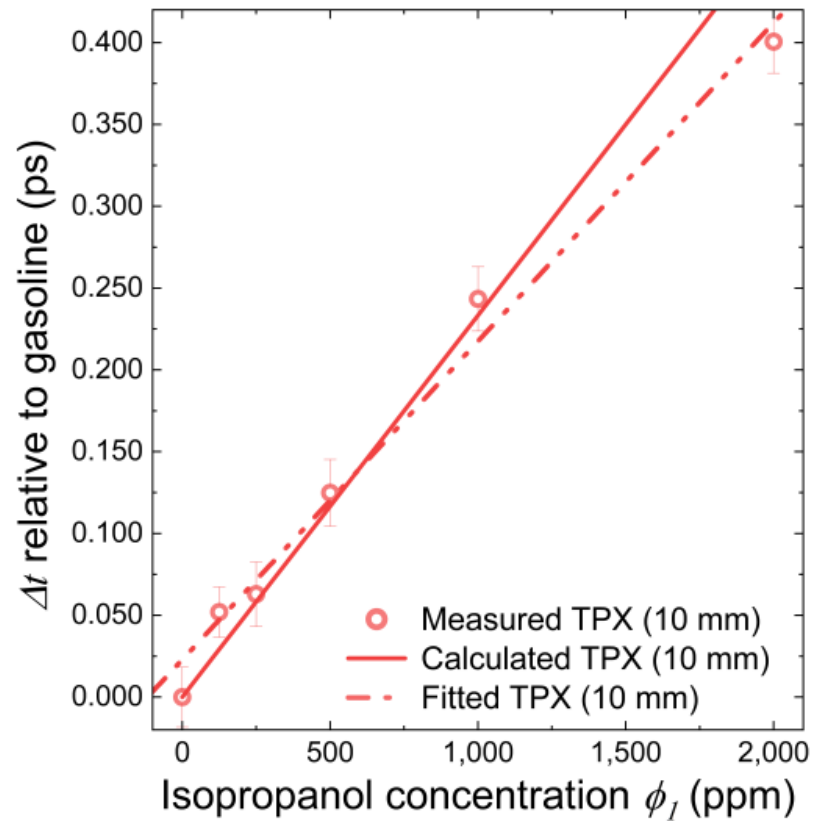

(a)

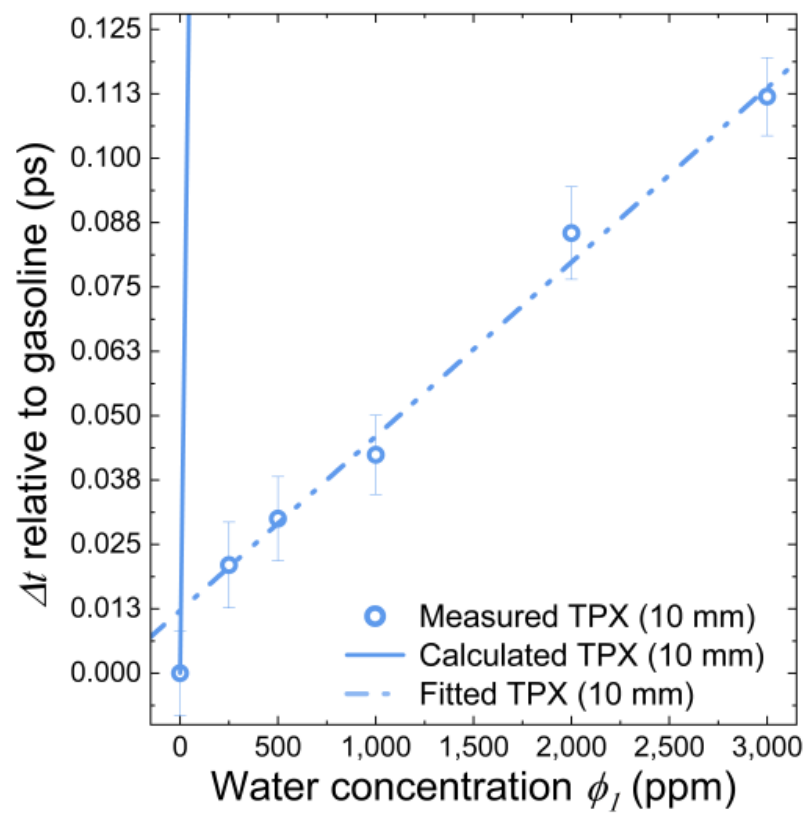

(b)

Figure 10. The THz pulse delays measured as the function of admixture concentrations by their mass fractions $\phi_{1}$ below 3000 ppm. (a) For isopropanol: $\phi_{1}=0,125,250,500,1000,2000$ ppm; (b) for water samples $\phi_{1}=0,250,500,1000,2000,3000 \mathrm{ppm}$. The solid lines represent theoretical dependence according to the Gladstone-Dale mixing rule given by Equation (11), and the dash-dotted lines represent the numerical fits. Note a good agreement between the theory and measurements in the case of isopropanol-gasoline admixtures and the discrepancy in the case of water-gasoline mixtures.

While a fairly good correspondence can be observed for isopropanol-gasoline samples, for which the measured pulse delays points are situated along the continuous red line, representing theoretical calculations, the agreement for water-containing samples cannot be confirmed. All measured points lay below the theoretical line and have substantially shorter TOFs than predicted by the calculations. Moreover, we have observed a reverse trend of decreasing pulse delays with an increasing water mass fraction above the $3000 \mathrm{ppm}$ 
mass fraction of water. All this suggests that a simple, quasi-binary mixture model of the refractive index is not applicable in this case.

It is well known that water is not miscible with gasoline. These two fractions will tend to separate depending on their relative concentrations in a mixture and environmental conditions $[4,40,41]$. Water forms complexes with gasoline containing liquid phase water and gasoline-water emulsions. Both fractions tend to separate, and the heavier water fraction sediments at the bottom of the cuvette by the presence of pure gasoline. This is why gasoline becomes a dominant fraction in the remaining volume of the liquid. Eventually, this trend causes the $\mathrm{THz}$ pulse to reverse because the incident radiation is exposed to a lower refractive index of gasoline. It is important to note that similar conclusions were drawn for other petrochemicals like diesel or crude oils emulsions as reported in $[13,14,42-45]$.

The fact that for water-gasoline mixtures, the delays of the THz pulses cannot be accurately predicted by ab initio calculations is of minor importance from the practical point of view of water detection. The empirically measured linear relation between $\Delta t$ and $\phi_{1}$ can still be used, providing an initial calibration with gasoline samples containing known concentrations of water is made before THz-TDS TOF measurements. We can, therefore, make use of this linearity and define the limits of detection of isopropanol and water admixtures in gasoline as the smallest measured mass fraction belonging to the linear trend of the measured data points. Based on this definition and looking at the graphs from Figure 10a,b, the detection limits of isopropanol and water were, respectively, $125 \mathrm{ppm}$ and $250 \mathrm{ppm}$. This satisfactory result obtained in a liquid phase approaches the detection limits of spectroscopic THz detection in a gas phase [46,47].

\section{Conclusions}

We have shown that a conceptually simple THz-TDS time-of-flight method relying on a straightforward $\mathrm{THz}$ pulse delay measurement can be successfully used to detect very small, on an order of parts-per-million of mass fraction, concentrations of water $\left(\mathrm{H}_{2} \mathrm{O}\right)$ or isopropanol $\left(\mathrm{C}_{3} \mathrm{H}_{8} \mathrm{O}\right)$ in quasi-binary gasoline solutions. The most important prerequisite for such sensitive detection is the stable time performance of a spectrometer and the femtosecond precision of the $\mathrm{THz}$ pulse time delay measurement.

The method is calibration-free, and ab initio calculations of the pulse delays resulting from its interaction with gasoline mixtures were possible, provided that a satisfactory model of the refractive index of a solution is known. We have found that this requirement is much better fulfilled for isopropanol-gasoline mixtures than for water-gasoline mixtures and attributed this fact to the good solubility of isopropanol in gasoline due to its alkyl group.

A modified model of the Gladstone-Dale mixing rule was used to represent the refractive index of gasoline mixtures. According to this model, the linear relation between the $\mathrm{THz}$ pulse propagation time and admixture mass fraction in a solution was found. This linear relation was qualitatively confirmed in the measurements. However, the quantitative agreement was found to be sufficiently satisfactory only in the case of isopropanol samples.

Based on the empirically measured linear relations between the pulse time of flight through the mixture and the mass fraction of water or isopropanol, we have determined the detection limits of these substances to be $250 \mathrm{ppm}$ and $125 \mathrm{ppm}$, respectively. These determined limits of sensitivity values are approaching those obtained with the use of much more complicated and time-consuming spectroscopic methods (like spectroscopy of the evaporated gasoline gas phase).

Therefore, our work clearly shows that the pulse time delay measurement by THz-TDS is a sensitive and fast method of gasoline contamination detection and, as such, can be easily integrated with industrial online real-time quality control applications.

Author Contributions: Conceptualization, K.S.; methodology, K.S. and E.K.-M.; software, K.S.; validation, K.S. and E.K.-M.; formal analysis, K.S.; investigation, K.S. and E.K.-M.; resources, T.S., and W.K. data curation, K.S. and E.K.-M.; writing—original draft preparation, K.S. and E.K.-M.; writing- 
review and editing, K.S., E.K.-M., and V.M., T.S. and W.K.; visualization, K.S.; funding acquisition, T.S., G.C. and W.K. All authors have read and agreed to the published version of the manuscript.

Funding: This work was supported by the "International Research Agendas" program of the Foundation for Polish Science co-financed by the European Union under the European Regional Development Fund (No. MAB/2018/9) for CENTERA.

Institutional Review Board Statement: Not applicable.

Informed Consent Statement: Not applicable.

Data Availability Statement: Not applicable.

Acknowledgments: We would like to thank B. Grzywacz for his technical assistance and PKN Orlen S. A. representatives for fruitful discussions.

Conflicts of Interest: The authors declare no conflict of interest. The funders had no role in the design of the study; in the collection, analyses, or interpretation of data; in the writing of the manuscript, or in the decision to publish the results.

\section{Appendix A}

This appendix gives formal derivations of the formulas that were used in Section 2.2.2. to estimate the Rayleigh lengths, $Z_{R}$, of the $\mathrm{THz}$ beams delivered to the samples by means of the $4 f$ and $2 f$ quasi-optical beam delivery setups.

In the Gaussian beam approximation, a beam waist, $\omega$, of a diverging one-dimensional beam with the intensity profile described by the Gaussian function is given by the formula [48]:

$$
\omega(z)=\omega_{0} \sqrt{1+\left(\frac{z}{Z_{R}}\right)^{2}} .
$$

The beam waist parameter describes the so-called Gaussian beam radius for which the intensity of the beam decreased to $1 / e^{2}$ of its axial or peak value. The origin of the coordinate system related to Equation (A1), $z=0$, is chosen at the point where the beam waist parameter is the smallest, corresponding to $\omega_{0}$. The distance $z=Z_{R}$, which is symmetrical on both positive and negative sides around $z=0$, defines another important parameter-the Rayleigh range, within which the beam waist does not increase above $\omega_{0} \sqrt{2}$. The $Z_{R}$ parameter is also known as the Rayleigh length. It is related to the beam waist, $\omega_{0}$, and the wavelength of the radiation, $\lambda$, by the following formula:

$$
Z_{R}=\frac{\pi\left(\omega_{0}\right)^{2}}{\lambda} .
$$

Inside the Rayleigh range, the beam is considered to be approximately collimated, and the propagating wave is approximated by the plane wave approximation. The halfdivergence angle of the beam outside the Rayleigh length, $\theta$, is given by:

$$
\theta=\frac{\lambda}{\pi \omega_{0}} .
$$

The above equation is valid for slowly diverging beams and uses the approximation $\tan (\theta) \approx \theta$.

Far beyond the Rayleigh range, a divergent Gaussian beam appears to have its origin at $z=0$. It propagates in the form of a radial wave. Since its source can be approximated by a point, the full divergence angle, $2 \theta$, can also be calculated by means of the geometrical optics as a diameter, $d$, illuminated on a mirror divided by the mirror's focal length, $f$ :

$$
2 \theta \approx \frac{d}{f}
$$


Therefore, a beam waist of a divergent $\mathrm{THz}$ wave and its diameter on a surface of a mirror shifted by a distance of the focal length from the $\mathrm{THz}$ emitter are related by the formula:

$$
\omega_{0}(\lambda) \approx \frac{2 \lambda f}{\pi d} .
$$

A THz pulse emitted by a photoconductive antenna of a THz-TDS system is a superposition of a wide range of wavelengths. According to Equation (A5), a diameter of each of these wavelength components at the input of an off-axis collimating mirror in a $4 f$ quasi-optical system (Figure 1a) can be calculated as:

$$
d(\lambda) \approx \frac{4 \lambda f_{\text {trans }}}{2 \omega_{\text {trans }} \pi},
$$

With $f_{\text {trans }}$ denoting the focal length of the mirror and $2 \omega_{\text {trans }}$ indicating the output aperture of Tx-transmitter, which is typically approximated by a diameter of a Si lens attached to the antenna to reduce the divergence of the beam. Note that the collimated $\mathrm{THz}$ beam propagates in the form of concentric cylinders, with the longer wavelengths being outside the shorter wavelengths. Such a beam is next focused by the second of the off-axis mirrors, and the wavelength-dependent beam waist in the focal spot can be calculated again by means of Equation (A5):

$$
\omega_{\text {focus }}(\lambda) \approx \frac{2 \lambda f_{\text {focus }}}{\pi d} .
$$

Here, $f_{\text {focus }}$ corresponds to the focal length of the focusing mirror and $\omega_{\text {focus }}$ corresponds to the focused beam waist. By inserting Equation (A6) into Equation (A7), $\omega_{\text {focus }}$ can be expressed in terms of the transmitted beam waist as:

$$
\omega_{\text {focus }} \approx \frac{f_{\text {focus }}}{f_{\text {trans }}} \omega_{\text {trans }} .
$$

A remarkable conclusion resulting from Equation (A8) is that despite substantially different divergences of the emitted $\mathrm{THz}$ pulse spectral components, the beam waists at the Fourier plane after focusing are the same. This distinctive attribute of a $4 f$ optical setup is not valid, e.g., for the $2 f$ system. The wavelength-independent beam pattern is again transformed to the wavelength-independent waist at the $\mathrm{THz}$ detector.

Given Equation (A8), it is possible to find the formula describing the Rayleigh lengths near the focus by substituting $\omega_{0}$ with $\omega_{\text {focus }}$ in Equation (A2) to finally obtain the following equation:

$$
Z_{R} \approx \frac{\pi}{\lambda}\left(\frac{f_{\text {focus }}}{f_{\text {trans }}} \omega_{\text {trans }}\right)^{2} .
$$

The estimation of Rayleigh lengths for the $2 f$ quasi-optical system (Figure 1b) can be made in a similar way. From the Gaussian beam theory it is known that a radiation source of a constant output aperture, $2 \omega_{\text {trans }}$, situated at a focal plane in front of a focusing mirror or lens of the focal length, $f_{\text {trans }}$, will form a wavelength-dependent profile of concentric collimated beams after the mirror. Within this profile, long-wavelength components lay concentrically outside the short-wavelength components. In this case, the wavelengthdependent beam waists at the coupling mirror can be calculated by means of Equation (A1) by putting $\omega_{0}=\omega_{\text {trans }}$ and $z=f_{\text {trans }}$.

The calculation of $Z_{R}$, which is present in this equation, again requires that $\omega_{0}=\omega_{\text {trans }}$. Knowing the beam waists, the wavelength-dependent Rayleigh lengths can be calculated according to Equation (A2). Note, however, that since the beam waists of the wavelength components at the coupling mirror are unequal in this case, the beam pattern in the Fourier plane consists of concentric circles with the radii of the longer wavelengths laying outside the radii of the shorter wavelengths. This means that the samples must be homogeneous 
over large areas in order to be investigated with the $2 f$ quasi-optical beam delivery systems. The estimations of Rayleigh lengths given in Section 2.2.2. were made using the methodology described above.

It is important to understand that, contrary to optical measurements, the THz-TDS measurements require careful designing of beam delivery setups and their parameters, including all optical elements and cuvette lengths. The paraxial Gaussian beam approximation, which in a strict mathematical formulation requires that $\omega_{0} \gg \lambda$, may not be fulfilled due to the sub-millimeter and millimeter wavelength components of the pulse. Still, it is a common practice to use Gaussian beam approximation in THz-TDS spectroscopy [49,50].

\section{Appendix B}

This appendix gives a formal derivation of Equation (11) from Section 2.4, which relates the $\mathrm{THz}$ pulse delay in admixture gasoline solution with respect to the pulse delay after propagation in pure gasoline based on the Gladstone-Dale mixing rule.

The equation describing the difference of THz pulse TOFs in isopropanol-gasoline solution and pure gasoline, $\Delta t$, the Gladstone-Dale mixing rule equation for the refractive index, $n_{D}$, of the isopropanol gasoline solution, and the relationship between the mass fractions of isopropanol, $\phi_{1}$, and gasoline, $\phi_{2}$, are given, respectively, by Equations (A10)-(A12) [32,33]:

$$
\begin{gathered}
\Delta t=t_{n_{D}}-t_{n_{D 2}}=\frac{l}{c}\left(n_{D}-1\right)-\frac{l}{c}\left(n_{D 2}-1\right)=\frac{l}{c}\left(n_{D}-n_{D 2}\right), \\
n_{D}-1=\phi_{1}\left(n_{D 1}-1\right)+\phi_{2}\left(n_{D 2}-1\right), \\
\phi_{1}+\phi_{2}=1 .
\end{gathered}
$$

Here, $l$ denotes the length of the cuvette, $c$ denotes the speed of light in vacuum, $n_{D 1}$ represents the refractive index of pure isopropanol, and $n_{D 2}$ indicates the refractive index of pure gasoline. Equation (A11) can be rewritten in the form:

$$
n_{D}-1=\phi_{1} n_{D 1}+\phi_{2} n_{D 2}-\left(\phi_{1}+\phi_{2}\right),
$$

which together with Equation (A12) yields:

$$
n_{D}=\phi_{1} n_{D 1}+\phi_{2} n_{D 2}
$$

The above equation was quoted in the main text as Equation (10). By inserting Equation (A14) into Equation (A10), one obtains:

$$
\begin{aligned}
& \Delta t=\frac{l}{c}\left(n_{D}-n_{D 2}\right)=\frac{l}{c}\left(\phi_{1} n_{D 1}+\phi_{2} n_{D 2}-n_{D 2}\right)=\ldots \\
& \ldots=\frac{l}{c}\left(\phi_{1} n_{D 1}+\phi_{2} \frac{\phi_{1}}{\phi_{1}} n_{D 2}-\frac{\phi_{1}}{\phi_{1}} n_{D 2}\right)=\frac{l}{c}\left(\phi_{1} n_{D 1}+\frac{\phi_{2} \phi_{1}-\phi_{1}}{\phi_{1}} n_{D 2}\right) .
\end{aligned}
$$

The fraction of the expression in the brackets can be expanded and, after the substitution of $\phi_{2}$ determined based on the Equation (A12), takes the form:

$$
\frac{\phi_{2} \phi_{1}-\phi_{1}}{\phi_{1}}=\frac{\left(1-\phi_{1}\right) \phi_{1}-\phi_{1}}{\phi_{1}}=\frac{\phi_{1}-\phi_{1} \phi_{1}-\phi_{1}}{\phi_{1}}=-\phi_{1},
$$

such that finally Equation (A15) can be rewritten to obtain the final formula for $\Delta t$ :

$$
\Delta t=\frac{l}{c}\left(\phi_{1} n_{D 1}+\frac{\phi_{2} \phi_{1}-\phi_{1}}{\phi_{1}} n_{D 2}\right)=\frac{l}{c}\left(\phi_{1} n_{D 1}-\phi_{1} n_{D 2}\right)=\frac{l}{c}\left(n_{D 1}-n_{D 2}\right) \phi_{1} .
$$

The difference of the THz pulse TOFs in gasoline with isopropanol and pure gasoline is proportional to the difference of the refractive indices of the dissolved substance and pure gasoline, the isopropanol concentration and the ratio of the cuvette length divided by the speed of light. Equation (A17) has the same form as Equation (11) from the main text. 


\section{References}

1. Schmitz, M.; Schmitz, G. Experimental Study on the Accretion and Release of Ice in Aviation Jet Fuel. Aerosp. Sci. Technol. 2018, 82-83, 294-303. [CrossRef]

2. Hemighaus, G.; Boval, T.; Bacha, J.; Barnes, F.; Franklin, M.; Gibbs, L.; Hogue, N.; Jones, J.; Lesnini, D.; Lind, J.; et al. Aviation Fuels Technical Review; Chevron Products Company, Chevron USA Inc.: San Ramon, CA, USA, 2007.

3. Baena-Zambrana, S.; Repetto, S.L.; Lawson, C.P.; Lam, J.K.-W. Behaviour of Water in Jet Fuel-A Literature Review. Prog. Aerosp. Sci. 2013, 60, 35-44. [CrossRef]

4. Zherebtsov, V.L.; Peganova, M.M. Water Solubility versus Temperature in Jet Aviation Fuel. Fuel 2012, 102, 831-834. [CrossRef]

5. Lam, J.K.-W.; Carpenter, M.D.; Williams, C.; Hetherington, J.; Lao, L.; Hammond, D.; Ramshaw, C.; Yeung, H. Water Behaviour in Aviation Fuel under Cold Temperature Conditions (WAFCOLT); European Aviation Safety Agency: Köln-Deutz, Germany, 2013.

6. Naftaly, M.; Vieweg, N.; Deninger, A. Industrial Applications of Terahertz Sensing: State of Play. Sensors 2019, 19, 4203. [CrossRef] [PubMed]

7. Ng, B.; Hanham, S.M.; Giannini, V.; Chen, Z.C.; Tang, M.; Liew, Y.F.; Klein, N.; Hong, M.H.; Maier, S.A. Lattice Resonances in Antenna Arrays for Liquid Sensing in the Terahertz Regime. Opt. Express 2011, 19, 14653-14661. [CrossRef]

8. Walther, M.; Fischer, B.M.; Ortner, A.; Bitzer, A.; Thoman, A.; Helm, H. Chemical Sensing and Imaging with Pulsed Terahertz Radiation. Anal. Bioanal. Chem. 2010, 397, 1009-1017. [CrossRef]

9. Yin, M.; Tang, S.; Tong, M. The Application of Terahertz Spectroscopy to Liquid Petrochemicals Detection: A Review. Appl. Spectrosc. Rev. 2016, 51, 379-396. [CrossRef]

10. Liu, L.; Pathak, R.; Cheng, L.-J.; Wang, T. Real-Time Frequency-Domain Terahertz Sensing and Imaging of Isopropyl AlcoholWater Mixtures on a Microfluidic Chip. Sens. Actuators B Chem. 2013, 184, 228-234. [CrossRef]

11. Arik, E.; Altan, H.; Esenturk, O. Dielectric Properties of Ethanol and Gasoline Mixtures by Terahertz Spectroscopy and an Effective Method for Determination of Ethanol Content of Gasoline. J. Phys. Chem. A 2014, 118, 3081-3089. [CrossRef]

12. Gorenflo, S.; Tauer, U.; Hinkov, I.; Lambrecht, A.; Buchner, R.; Helm, H. Dielectric Properties of Oil-Water Complexes Using Terahertz Transmission Spectroscopy. Chem. Phys. Lett. 2006, 421, 494-498. [CrossRef]

13. Jin, W.-J.; Zhao, K.; Yang, C.; Xu, C.-H.; Ni, H.; Chen, S.-H. Experimental Measurements of Water Content in Crude Oil Emulsions by Terahertz Time-Domain Spectroscopy. Appl. Geophys. 2013, 10, 506-509. [CrossRef]

14. Guan, L.; Zhan, H.; Miao, X.; Zhu, J.; Zhao, K. Terahertz-Dependent Evaluation of Water Content in High-Water-Cut Crude Oil Using Additive-Manufactured Samplers. Sci. China Phys. Mech. Astron. 2017, 60, 044211. [CrossRef]

15. Specification of Unleaded 95-Octane Gasoline, PKN ORLEN. Available online: https://www.orlen.pl/en/for-business / products / fuels/petrol/unleaded-95 (accessed on 2 January 2022).

16. Ahmed, S.M.; Ahmad, F.; Osman, S.M. Preparation and Characterization of Derivatives of Isoricinoleic Acid and Their Antimicrobial Activity. J. Am. Oil Chem. Soc. 1985, 62, 1578-1580. [CrossRef]

17. Mercier, L.; Detellier, C. Preparation, Characterization, and Applications as Heavy Metals Sorbents of Covalently Grafted Thiol Functionalities on the Interlamellar Surface of Montmorillonite. Environ. Sci. Technol. 1995, 29, 1318-1323. [CrossRef]

18. Amer, A.; Mehlhorn, H. Larvicidal Effects of Various Essential Oils against Aedes, Anopheles, and Culex Larvae (Diptera, Culicidae). Parasitol. Res. 2006, 99, 466-472. [CrossRef]

19. Dietz, R.J.B.; Vieweg, N.; Puppe, T.; Zach, A.; Globisch, B.; Göbel, T.; Leisching, P.; Schell, M. All Fiber-Coupled THz-TDS System with kHz Measurement Rate Based on Electronically Controlled Optical Sampling. Opt. Lett. 2014, 39, 6482-6485. [CrossRef]

20. Auston, D.H. Chapter 4-Picosecond Photoconductors: Physical Properties and Applications. In Picosecond Optoelectronic Devices; Lee, C.H., Ed.; Academic Press: Cambridge, MA, USA, 1984; pp. 73-117. ISBN 978-0-12-440880-7.

21. Tani, M.; Matsuura, S.; Sakai, K.; Nakashima, S. Emission Characteristics of Photoconductive Antennas Based on LowTemperature-Grown GaAs and Semi-Insulating GaAs. Appl. Opt. 1997, 36, 7853-7859. [CrossRef] [PubMed]

22. Dietz, R.J.B.; Globisch, B.; Gerhard, M.; Velauthapillai, A.; Stanze, D.; Roehle, H.; Koch, M.; Göbel, T.; Schell, M. 64 W Pulsed Terahertz Emission from Growth Optimized InGaAs/InAlAs Heterostructures with Separated Photoconductive and Trapping Regions. Appl. Phys. Lett. 2013, 103, 061103. [CrossRef]

23. Dietz, R.J.B.; Globisch, B.; Roehle, H.; Stanze, D.; Göbel, T.; Schell, M. Influence and Adjustment of Carrier Lifetimes in InGaAs/InAlAs Photoconductive Pulsed Terahertz Detectors: $6 \mathrm{THz}$ Bandwidth and 90dB Dynamic Range. Opt. Express 2014, 22, 19411-19422. [CrossRef]

24. Vieweg, N.; Rettich, F.; Deninger, A.; Roehle, H.; Dietz, R.; Göbel, T.; Schell, M. Terahertz-Time Domain Spectrometer with 90 DB Peak Dynamic Range. J. Infrared Millim. Terahertz Waves 2014, 35, 823-832. [CrossRef]

25. Jepsen, P.U.; Cooke, D.G.; Koch, M. Terahertz Spectroscopy and Imaging-Modern Techniques and Applications. Laser Photonics Rev. 2011, 5, 124-166. [CrossRef]

26. Klingshirn, C.F. Maxwell's Equations, Photons and the Density of States. In Semiconductor Optics; Klingshirn, C.F., Ed.; Graduate Texts in Physics; Springer: Berlin/Heidelberg, Germany, 2012; pp. 11-37. ISBN 978-3-642-28362-8.

27. Pretorius, F.; Focke, W.W.; Androsch, R.; du Toit, E. Estimating Binary Liquid Composition from Density and Refractive Index Measurements: A Comprehensive Review of Mixing Rules. J. Mol. Liq. 2021, 332, 115893. [CrossRef]

28. Tasic, A.Z.; Djordjevic, B.D.; Grozdanic, D.K.; Radojkovic, N. Use of Mixing Rules in Predicting Refractive Indexes and Specific Refractivities for Some Binary Liquid Mixtures. J. Chem. Eng. Data 1992, 37, 310-313. [CrossRef] 
29. Resa, J.M.; González, C.; Ortiz de Landaluce, S.; Lanz, J. Densities, Excess Molar Volumes, and Refractive Indices of Ethyl Acetate and Aromatic Hydrocarbon Binary Mixtures. J. Chem. Thermodyn. 2002, 34, 995-1004. [CrossRef]

30. Krishnaswamy, R.K.; Janzen, J. Exploiting Refractometry to Estimate the Density of Polyethylene: The Lorentz-Lorenz Approach Re-Visited. Polym. Test. 2005, 24, 762-765. [CrossRef]

31. Iglesias-Otero, M.A.; Troncoso, J.; Carballo, E.; Romaní, L. Density and Refractive Index in Mixtures of Ionic Liquids and Organic Solvents: Correlations and Predictions. J. Chem. Thermodyn. 2008, 40, 949-956. [CrossRef]

32. Nita, I.; Iulian, O.; Geacai, E.; Osman, S. Physico-Chemical Properties of the Pseudo-Binary Mixture Gasoline $+1-$ Butanol. Energy Procedia 2016, 95, 330-336. [CrossRef]

33. Nita, I.; Geacai, E.; Iulian, O.; Osman, S. Study of the Refractive Index of Gasoline+alcohol Pseudo-Binary Mixtures. Ovidius Univ. Ann. Chem. 2017, 28. [CrossRef]

34. Arik, E.; Altan, H.; Esenturk, O. Dielectric Properties of Diesel and Gasoline by Terahertz Spectroscopy. J. Infrared Millim. Terahertz Waves 2014, 35, 759-769. [CrossRef]

35. Bettega, M.H.F.; Winstead, C.; McKoy, V.; Jo, A.; Gauf, A.; Tanner, J.; Hargreaves, L.R.; Khakoo, M.A. Collisions of Low-Energy Electrons with Isopropanol. Phys. Rev. A 2011, 84, 042702. [CrossRef]

36. Lapuerta, M.; Rodríguez-Fernández, J.; Patiño-Camino, R.; Cova-Bonillo, A.; Monedero, E.; Meziani, Y.M. Determination of Optical and Dielectric Properties of Blends of Alcohol with Diesel and Biodiesel Fuels from Terahertz Spectroscopy. Fuel 2020, 274, 117877. [CrossRef]

37. Huang, S.; Ashworth, P.C.; Kan, K.W.C.; Chen, Y.; Wallace, V.P.; Zhang, Y.; Pickwell-MacPherson, E. Improved Sample Characterization in Terahertz Reflection Imaging and Spectroscopy. Opt. Express 2009, 17, 3848-3854. [CrossRef]

38. Jin, Y.-S.; Kim, G.-J.; Shon, C.-H.; Jeon, S.-G.; Kim, J.I. Analysis of Petroleum Products and Their Mixtures by Using Terahertz Time Domain Spectroscopy. J. Korean Phys. Soc. 2008, 53, 1879-1885. [CrossRef]

39. Al-Douseri, F.M.; Chen, Y.; Zhang, X.-C. THz Wave Sensing for Petroleum Industrial Applications. Int. J. Infrared Millim. Waves 2006, 27, 481-503. [CrossRef]

40. Gramajo de Doz, M.B.; Bonatti, C.M.; Sólimo, H.N. Water Tolerance and Ethanol Concentration in Ethanol-Gasoline Fuels at Three Temperatures. Energy Fuels 2004, 18, 334-337. [CrossRef]

41. Thanikasalam, K.; Rahmat, M.; Fahmi, A.G.M.; Zulkifli, A.M.; Shawal, N.N.; Ilanchelvi, K.; Ananth, M.; Elayarasan, R. A Review of Phase Separation Issues in Aviation Gasoline Fuel and Motor Gasoline Fuels in Aviation. IOP Conf. Ser. Mater. Sci. Eng. 2018, 370, 012007. [CrossRef]

42. Kwon, G.; Kota, A.K.; Li, Y.; Sohani, A.; Mabry, J.M.; Tuteja, A. On-Demand Separation of Oil-Water Mixtures. Adv. Mater. 2012, 24, 3666-3671. [CrossRef] [PubMed]

43. Song, Y.; Zhan, H.L.; Zhao, K.; Miao, X.Y.; Lu, Z.Q.; Bao, R.M.; Zhu, J.; Xiao, L.Z. Simultaneous Characterization of Water Content and Distribution in High-Water-Cut Crude Oil. Energy Fuels 2016, 30, 3929-3933. [CrossRef]

44. Feng, X.; Wu, S.-X.; Zhao, K.; Wang, W.; Zhan, H.-L.; Jiang, C.; Xiao, L.-Z.; Chen, S.-H. Pattern Transitions of Oil-Water Two-Phase Flow with Low Water Content in Rectangular Horizontal Pipes Probed by Terahertz Spectrum. Opt. Express 2015, 23, A1693-A1699. [CrossRef]

45. Palchoudhury, S.; Lead, J.R. A Facile and Cost-Effective Method for Separation of Oil-Water Mixtures Using Polymer-Coated Iron Oxide Nanoparticles. Environ. Sci. Technol. 2014, 48, 14558-14563. [CrossRef]

46. Hepp, C.; Lüttjohann, S.; Roggenbuck, A.; Deninger, A.; Nellen, S.; Göbel, T.; Jörger, M.; Harig, R. A Cw-Terahertz Gas Analysis System with ppm Detection Limits. In Proceedings of the 2016 41st International Conference on Infrared, Millimeter, and Terahertz waves (IRMMW-THz), Copenhagen, Denmark, 25-30 September 2016; pp. 1-2.

47. Nellen, S.; Globisch, B.; Kohlhaas, R.B.; Liebermeister, L.; Schell, M. Recent Progress of Continuous-Wave Terahertz Systems for Spectroscopy, Non-Destructive Testing, and Telecommunication. In Proceedings of the Terahertz, RF, Millimeter, and Submillimeter-Wave Technology and Applications XI, San Francisco, CA, USA, 29 January-1 February 2018; SPIE: Bellingham, WA, USA, 2018; Volume 10531, pp. 44-51.

48. Dexheimer, S.L. Terahertz Spectroscopy: Principles and Applications; CRC Press: Boca Raton, 2008; ISBN 978-0-8493-7525-5.

49. Peiponen, K.-E.; Zeitler, A.; Kuwata-Gonokami, M. (Eds.) Terahertz Spectroscopy and Imaging; Springer Series in Optical Sciences; Springer: Berlin/Heidelberg, Germany, 2013; ISBN 978-3-642-29563-8.

50. Ahi, K. Mathematical Modeling of THz Point Spread Function and Simulation of THz Imaging Systems. IEEE Trans. Terahertz Sci. Technol. 2017, 7, 747-754. [CrossRef] 